\title{
Overcoming Multiple Absorption Barrier for Insulin Oral Delivery Using Multifunctional Nanoparticles Based on Chitosan Derivatives and Hyaluronic Acid
}

This article was published in the following Dove Press journal:

International Journal of Nanomedicine

\author{
Zuxian Chen ${ }^{1, *}$ \\ Shangcong $\mathrm{Han}^{1}{ }^{1} *$ \\ Xiaotang Yang' \\ Lisa $X u^{1}$ \\ Hong $\mathrm{Qi}^{2}$ \\ Guizhou $\mathrm{HaO}^{3}$ \\ Jie Cao ${ }^{\prime}{ }^{\prime}$ \\ Yan Liang' \\ Qingming $\mathrm{Ma}^{\mathrm{I}}$ \\ Guimin Zhang ${ }^{3}$ \\ Yong Sun (D)
}

'Department of Pharmaceutics, School of Pharmacy, Qingdao University, Qingdao 26602I, People's Republic of China;

${ }^{2}$ Department of General Surgery, Qingdao Municipal Hospital, Qingdao 26607I, People's Republic of China; ${ }^{3}$ Department of Scientific Research, Lunan Pharmaceutical Corporation, Linyi 27600I, People's Republic of China

*These authors contributed equally to this work
Background: Although dynamics and uses of modified nanoparticles (NPs) as orally administered macromolecular drugs have been researched for many years, measures of molecule stability and aspects related to important transport-related mechanisms which have been assessed in vivo remain as relatively under characterized. Thus, our aim was to develop a novel type of oral-based delivery system for insulin and to overcome barriers to studying the stability, transport mechanisms, and efficacy in vivo of the delivery system.

Methods: NPs we developed and tested were composed of insulin (INS), dicyandiamidemodified chitosan (DCDA-CS), cell-penetrating octaarginine (r8), and hydrophilic hyaluronic acid (HA) and were physically constructed by electrostatic self-assembly techniques.

Results: Compared to free-insulin, levels of HA-DCDA-CS-r8-INS NPs were retained at more desirable measures of biological activity in our study. Further, our assessments of the mechanisms for NPs suggested that there were high measures of cellular uptake that mainly achieved through active transport via lipid rafts and the macropinocytosis pathway. Furthermore, investigations of NPs indicated their involvement in caveolae-mediated transport and in the DCDA-CS-mediated paracellular pathway, which contributed to increasing the efficiency of sequential transportation from the apical to basolateral areas. Accordingly, high efficiency of absorption of NPs in situ for intestinal loop models was realized. Consequently, there was a strong induction of a hypoglycemic effect in diabetic rats of NPs via orally based administrations when compared with measures related to free insulin. Conclusion: Overall, the dynamics underlying and influenced by HA-DCDA-CS-r8-INS may hold great promise for stability of insulin and could help overcome interference by the epithelial barrier, and thus showing a great potential to improve the efficacy of orally related treatments.

Keywords: insulin, oral drug delivery, transepithelial transport, paracellular pathway, caveolae-mediated transport

\section{Introduction}

Insulin has been the primarily used drug for patients afflicted with both insulindependent and non-insulin-dependent diabetes mellitus. However, accounts of harm to patients have been reported from courses of treatments with injectable types of insulin preparations. ${ }^{1}$ Many potential and realized side effects from subcutaneous and intravenous treatments occur, including pain and fat atrophy at injection sites among
Correspondence: Yong Sun $\mathrm{Tel}+86-532-8299 \mid 203$

Email sunyong@qdu.edu.cn 


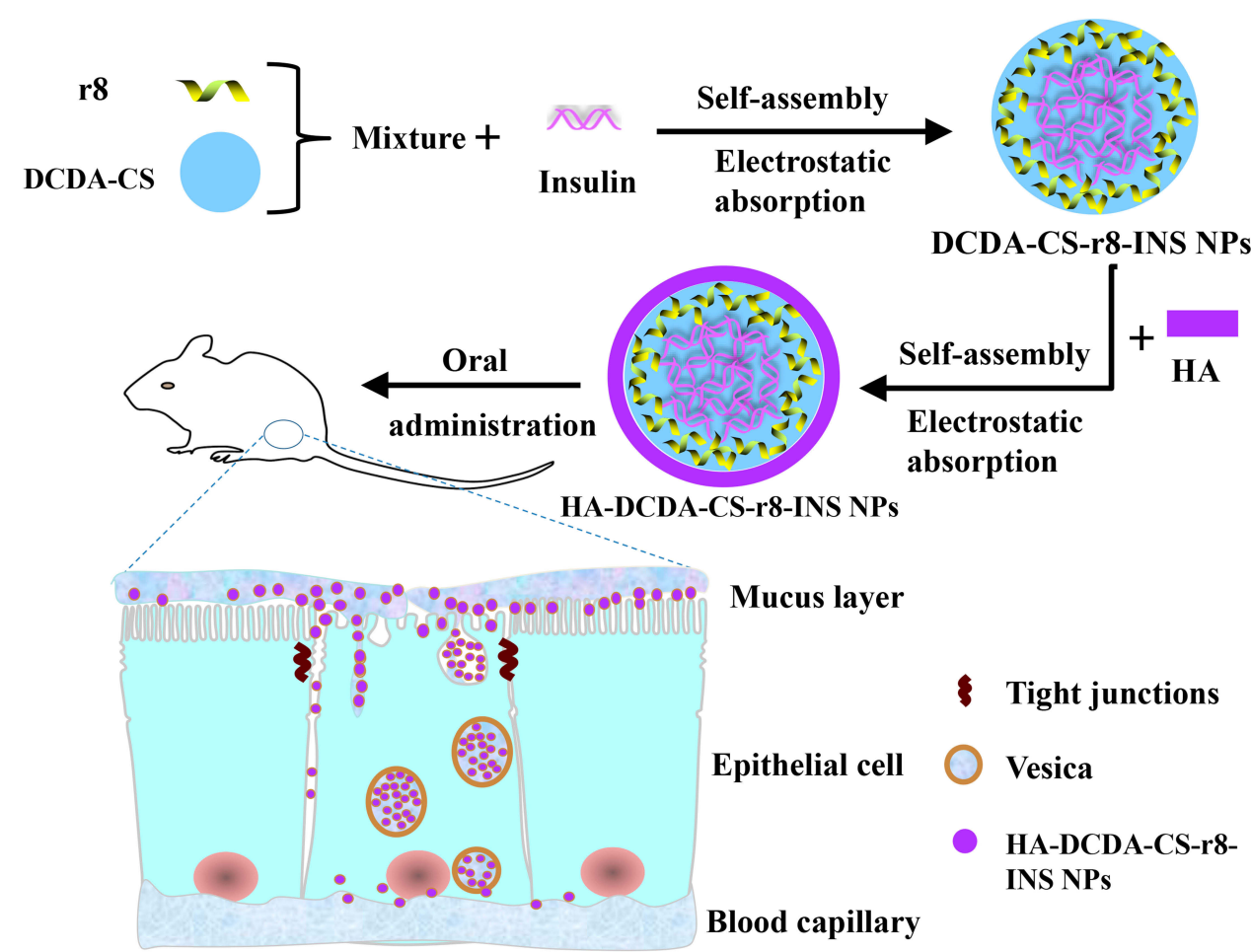

Figure I Schematic diagram of preparation process of HA-DCDA-CS-r8-INS NPs and transepithelial transport in the intestinal epithelium.

other undesirable effects, ${ }^{2}$ thus, effective non-injectable types of treatments remain desirable. Among non-injectable insulin preparations, orally consumed forms have high convenience and relatively high levels of patient compliance. ${ }^{3}$ Furthermore, orally consumed forms may help to induce desirable stimulation of physiological secretions of the pancreas. These secretions consequently allow insulin to enter the liver through the hepatic portal vein thereafter entering peripheral tissues, ultimately resulting in beneficial reductions in concentration-based blood sugar and hypoglycemia risk across the entire circulatory system. ${ }^{4}$ Therefore, oral forms are possibly ideal means for insulin delivery and have become an optimal choice for many patients. ${ }^{5}$

However, challenges exist despite some of the unique advantages of orally applied insulin. For example, a protein/ peptide drug delivered orally plays an effective role as a pharmacodynamic only upon passing into intestinal epithelium, out of the intestines, and then back into blood circulation. ${ }^{6}$ During these and related processes, insulin becomes chemically degraded and broken down through exposure to proteases which are relatively abundant in gastrointestinal tracts. Mucus adhered intestinal epithelial cells act to trap and remove pathogens, and remove foreign particles, especially including cationic substances. ${ }^{7}$ Thus, assessment of the effectiveness of large-sized molecular- based drugs orally consumed to consequent eventual presence in epithelial related cells is challenging. ${ }^{8}$ Accordingly, it becomes rather awkward to make insulin to penetrate into intestinal epithelial layers because of the low permeability of epithelial cells for hydrophilic macromolecular structured drugs. In addition, intercellular tight junctions can act such as to block paracellular transport of insulin. Likewise, the pathway by which nano-based drugs enter on the basolateral side, and then are released to the bloodstream has been assessed, but has thus far been found to be quite unpredictable.

To pass through biological barriers that exist and which could limit the applications and efficacy of oral delivery of insulin, varied delivery systems have been developed. For example, natural polymer nanoparticles, synthetic polymer nanoparticles, solid lipid nanoparticles, liposomes, nanoemulsions, as well as inorganic nanoparticles have been examined. ${ }^{9}$ Nano-drug delivery systems induced the protection of peptides and transited cargo across mucus layers and into intestinal epithelial cells. ${ }^{10,11}$ Furthermore, heightened oral bioavailability of insulin could have been achieved in relation to chemical-based modifications, endowing functionality to ligands, and through the modification of cell-penetrating peptides for insulin and enzyme inhibitors. ${ }^{12}$ Recent studies have shown that 
L-valine modified chitosan nanoparticles have great potential in oral insulin delivery. ${ }^{13}$ Choline and geranate (CAGE) ionic liquid-based oral insulin formulation enhanced paracellular transport of insulin. ${ }^{14}$ And deoxycholic acid-modified nanoparticles (DNPs) exploited the bile acid pathway to effectively overcome barriers of the intestinal epithelium. ${ }^{15}$ Morishita and colleagues presented results which indicated that cell-penetrating peptides had the ability to help promote intestinal absorption of insulin. $^{12}$ In fact, overcoming barrier in the intestinal epithelium has been the focus of research. Nevertheless, due to the existence of multiple types of biological, enzymatic, mucus, and epithelial oriented cellular barriers and variations in the dynamics underlying them, an entirely effective and accurate oral-based insulin delivery system has not yet been realized.

In the last decade, chitosan (CS) and its derivatives have been widely studied with respect to oral delivery of insulin as they are relatively non-toxic, biocompatible, and have a defined macromolecular structure. ${ }^{16}$ Nanoparticles based upon chitosan have the ability to open tight junctions reversibly across the paracellular pathway, and are thus able to escape from the intricate dynamics of breakdown during intracellular transport. ${ }^{17}$ And it's been reported that using amphiphilic polymer n-octyl-n-arginine-chitosan, ${ }^{18}$ arginine-modified chitosan (CS-N-Arg) ${ }^{19}$ or amphiphilic chitosan derivative and $\operatorname{arginine}^{20}$ as carriers can enhance the transport of the drug across intestinal epithelial cell layer. In practice, chitosan has been one of the most optimal materials used for the application of oral insulin. However, CS does not dissolve in water but rather dissolves when among inorganic or organic acid. ${ }^{21}$ Thus, it is difficult for CS to electrostatically self-assemble with insulin in the absence of chemical crosslinkers. ${ }^{22}$ Therefore, we sought to synthesize dicyandiamide chitosan (DCDA-CS) to improve the water solubility of chitosan, resulting in realization preparation of insulin nanoparticles in neutral condition and simplify steps in the preparation process for orally consumed insulin.

While the use CS and NPs would be expected to alleviate some concerns, the hydrophobicity provided by cationic materials decreases permeation of mucus by nanoparticles. Whereupon, we used HA as the external hydrophilic electronegative coating for NPs. Because 1) hyaluronic acid (HA), which has outstanding biocompatibility, biodegradability, and low toxicity, exists in extracellular matrices and synovial fluids in most human tissues; ${ }^{23}$ 2) studies have shown that NPs with hydrophilic surface properties are inclined to penetrate mucus; ${ }^{24} 3$ ) hyaluronic acid adhered to intestinal epithelial cells may be a good candidate for facilitating carrier interactions with the intestinal wall. ${ }^{25}$ What is more, to achieve effective absorption, NPs must cross barriers of mucus and intestinal epithelial cells in an ordered sequence. Cellpenetrating peptides (CPPs) are short peptides, which promote cell internalization of various molecular carriers, ${ }^{26}$ facilitate trans-epithelial transport of different proteins in the form of covalent bonds or physical mixtures. ${ }^{27}$ Moreover, Kamei and colleagues ${ }^{28}$ verified the electrostatic interaction between CPP and the drug, and they found that $r 8$ enhanced the intestinal absorption of insulin, gastrin and GLP.

In this study, we thus sought to develop polymer nanoparticles for use in oral-based delivery to manage insulin and use multifunctional-based analyses to assess the efficacy of insulin nanoparticles (HA-DCDA-CS-r8-INS NPs) (Figure 1). The NPs possess core insulin coated with $\mathrm{r} 8$ and chitosan-based polymers (DCDA-CS), and a hydrophilic coating of hyaluronic acid by electrostatic interactions and such as to maintain gastrointestinal stability. Moreover, the addition of octaarginine (r8) in NPs may be used to help improve the penetrability and transepithelial transport of the insulin-related nanoparticles. Simultaneously, the inclusion of DCDA-CS was expected to help facilitate the opening of tight junctions whereby NPs could then be transported into the bloodstream via the paracellular pathway. We hoped that our approach would help facilitate better measures of molecular stability, permeability, and transintestinal epithelial absorption.

\section{Materials and Methods Materials}

Chitosan (Average $\mathrm{Mw}=380 \mathrm{kDa}$, deacetylation degree $=$ 90.2\%) was purchased from Shandong Aokang Biotechnology co. LTD. Potassium persulfate and dicyandiamide were purchased from MACKLIN. DCDA-CS was made from chemical constituents in our own laboratory. Hyaluronic acid (HA, 10kDa) was purchased from Meilun Biotechnology Co., Ltd. (China). Porcine insulin was purchased from Wanbang Bio-Chemical Co., Ltd. (Jiangsu, China). Octaarginine (r8) was chemically synthesized by Jill Biochemical. FITC-Insulin was synthesized by Beijing Hongyu Co., Ltd. (China). Tetramethyl rhodamine isothiocyanate (TRITC) was purchased from Beijing Brinway Technology Co., Ltd. (China). 3- (4, 5-dimethyl-2-thiazolium) 
-2, 5-diphenyl-2-H-tetrazolium bromide (MTT), Hoechst 33,342, trypsin, and pepsin were purchased from Beijing Solarbio Science and Technology Co., Ltd. (China). Genistin, chlorpromazine, and amiloride were all purchased from Sigma-Aldrich (St. Louis, MO, USA). Streptozotocin purchased was from G-clone Beijing Biotechnology Technology Co., Ltd., ABT650 (China). The Porcine INS ELISA kit was purchased from Nanjing Jiancheng Bioengineering Institute (China). Caco-2 cells were purchased from Shanghai Gefan Biotechnology. C0., Ltd. (China). All other chemical reagents in the study and those used to create in house reagents were confirmed as analytical grade.

\section{Synthesis and Characterization of DCDA-CS}

Graft copolymerizations proceeded in a three-necked bottle with a thermometer, reflux condenser, and magnetic stirrer. ${ }^{21}$ Chitosan ( $1.0 \mathrm{~g})$ was dissolved in acetic acid $(1 \% \mathrm{v} / \mathrm{v})$, stirred continuously at a constant temperature of $60^{\circ} \mathrm{C}$. Then, the prepared potassium persulfate solution of $5 \mathrm{~mL}(0.74 \mathrm{mM})$ was added to the three-necked bottle. Next, $50 \mathrm{~mL}$ of DCDA $(40 \mathrm{mM})$ was added in a dropwise fashion. The reaction was allowed to continue for $2 \mathrm{hrs}$, whereupon we followed this with 20 mins of stirring at room temperature. The reaction product was then precipitated by isopropanol $(250 \mathrm{~mL})$, was filtered, and was washed such as to remove the unmodified and relatively soluble and low-molecular weight components mostly made up of chitosan. The final grafted polymer was evaporated at $60^{\circ} \mathrm{C}$ and the concentrated solution was dialyzed at room temperature for 3 days. The solutions were then freeze-dried in order to obtain a solidified powder of DCDA-CS. In addition, we performed infrared spectroscopic analyses for DCDA-CS by using infrared spectrometry (Nicolet iS50, Thermo Fisher Scientific, USA). Solubility tests $^{21}$ for DCDA-CS were undertaken as follows: respectively, CS or DCDA-CS were dissolved in water, hydrochloric acid, 1\% acetic acid, dimethyl sulfoxide, tetrahydrofuran, acetone, and isopropanol at the concentration of $1 \mathrm{mg} / \mathrm{mL}$ at $60^{\circ} \mathrm{C}$ with magnetic stirring for $30 \mathrm{mins}$.

\section{Preparation of Insulin Nanoparticles}

Optimization of preparation conditions was as follows. DCDA-CS-INS NPs were prepared by setting different $\mathrm{pH}$ values of insulin solution and different mass ratios of DCDA-CS to insulin, wherein we accounted for particle size, encapsulation efficiency, and drug loading as optimization indexes.
HA-DCDA-CS-r8-INS NPs were prepared through a two-step preparation by way of using electrostatic selfassembly. $^{24}$ Briefly, we dissolved insulin in $0.01 \mathrm{~mol} / \mathrm{L}$ hydrochloric acid at a concentration of $1 \mathrm{mg} / \mathrm{mL}$ and the $\mathrm{pH}$ was adjusted to 7.0 by way of adding $1 \mathrm{~mol} / \mathrm{L} \mathrm{NaOH}$. In addition, we used $0.5 \mathrm{mg}$ of DCDA-CS which was dissolved in $1 \mathrm{~mL}$ of deionized water and was held at $60^{\circ} \mathrm{C}$ in a water bath for $30 \mathrm{~min}$. Octaarginine was then dissolved in deionized water at a concentration of $1 \mathrm{mg} /$ $\mathrm{mL}$. In order to prepare DCDA-CS-r8-INS NPs, we added insulin solution dropwise to the mixture and system containing octaarginine and DCDA-CS under constant magnetic stirring for $10 \mathrm{~min}$. Then, hyaluronic acid was dissolved in deionized water at a concentration of $0.5 \mathrm{mg} / \mathrm{mL}$. DCDA-CS-r8-INS NPs were then added in a dropwise fashion to the HA solution with constant magnetic-based agitation for $10 \mathrm{~min}$. The HA-DCDA-CS-r8INS NPs were prepared successfully in a fashion that produced a slightly blue color. After the step of freezedrying, HA-DCDA-CS-r8-INS NPs were available for use in an easily measurable powder form.

\section{Characterization of Insulin Nanoparticles}

Measures of particle size, particle dispersion, and Zetapotential for insulin nanoparticles were carried out for experiments at $25^{\circ} \mathrm{C}$. Experiments were performed in triplicate using a Malvern Zetasize NanoZS90 (British Malvern Instrument Co. Ltd). The morphology of insulin nanoparticles was observed by transmission electron microscopy (TEM, JSM-6490LA, Japanese company JEOL). To evaluate measures of encapsulated efficiency (EE \%) and drug loading (DL \%), the nanoparticles in powder form were dissolved in deionized water and suspensions of NPs were centrifuged at 13, $000 \mathrm{rpm}$ for $30 \mathrm{~min}$ at $4^{\circ} \mathrm{C} .{ }^{29}$ After centrifugation, the amounts of insulin in supernatant were measured by way of highperformance liquid chromatography (HPLC, Agilent 1200 Series). ${ }^{30}$ The mobile phase was composed of $0.1 \mathrm{~mol} / \mathrm{L}$ sodium dihydrogen phosphate solution ( $\mathrm{pH}$ adjusted to 3.0 by use of phosphoric acid): acetonitrile (72:28) and the detection wavelength was $214 \mathrm{~nm}$. The chromatographic column that we used was the ZORBAX SB-C18 $(4.6 \times 150 \mathrm{~mm}, 5 \mu \mathrm{m})$. The injection volume that we used was $20 \mu \mathrm{L}$ and the column temperature was $40^{\circ} \mathrm{C}$. And the insulin standard curve related to the calculation of insulin content was shown in Figure S1. The formulas for calculation of encapsulated efficiency (EE \%) and drug loading efficiency (DL \%) were as follows: 


$$
\begin{aligned}
& \text { Amount of insulin added - Amount of insulin } \\
& E E \%=\frac{\text { in the supernatant }}{} \\
& \text { Amount of insulin added - Amount of insulin } \\
& D L \%=\frac{\text { in the supernatant }}{\times 100 \%}
\end{aligned}
$$

\section{Integrity Study of Insulin Nanoparticles}

To verify whether or not insulin was successfully encapsulated in NPs, measures of interactions between DCDA-CS and insulin were analyzed by the use of fluorescence resonance energy transfer (FRET) methodologies. ${ }^{31}$ DCDA-CS was labeled with TRITC as a receptor and insulin was labeled with FITC as a donor, and fluorescent-labeled nanoparticles were prepared using the methods mentioned above. Fluorescently labeled NPs (equivalent to $10 \mu \mathrm{g} / \mathrm{mL}$ insulin) were excited at a wavelength of 490 or $550 \mathrm{~nm}$ and emission spectra were recorded at 500 700 nm using fluorescencebased spectrophotometry (F-4600, HITACHI). The formulas we used to calculate measures of FRET efficiency (E) and the distance between the donor and receptor $(\mathrm{R})$ were as follows:

$$
\begin{gathered}
E=1-\frac{F D A}{F D} \\
R=R 0-\sqrt[6]{\left(\frac{1}{E}-1\right)}
\end{gathered}
$$

where $\mathrm{F}_{\mathrm{DA}}$ is the intensity in the presence of the acceptor, $\mathrm{F}_{\mathrm{D}}$ is the intensity in the absence of the acceptor, and $\mathrm{R}_{0}$ is the Forster distance at $50 \%$ transfer efficiency. For FITC-TRITC, $\mathrm{R}_{0}$ is equivalent to a value of $55 \mathrm{~nm}$.

\section{Investigation of the Stability of Insulin Nanoparticles}

Insulin solutions and insulin NPs were put into simulated gastric fluid ( $\mathrm{SGF}, \mathrm{pH}=2.0$, pepsin) or simulated intestinal fluid ( $\mathrm{SIF}, \mathrm{pH}=6.8$, trypsin) respectively, at $37^{\circ} \mathrm{C}$. We then placed samples upon a shaking table set to $100 \mathrm{r} / \mathrm{min}$ and set to run for a pre-specified time. ${ }^{4}$ In addition, $400 \mu \mathrm{L}$ of sample was collected from SGF or SIF at predetermined time points and placed into a quartz cell. The corresponding values for the spectrum were recorded at $20^{\circ} \mathrm{C}$ by the use of a Chirascan spectrophotometer (Chirascan, Applied Photophysics, Surrey, UK). We used a correspondingly set scanning speed of $1 \mathrm{~nm} / \mathrm{s}$ and determined measures at wavelengths from $180 \mathrm{~nm}$ to $260 \mathrm{~nm}$. Then, baseline values for sample background were recorded and subtracted from the curves for the respective different samples by use of the 6-point smoothing technique. Furthermore, we used CDNN-based analyses to perform assessments of the influence of the spectrum and to analyze the contribution of different protein secondary structures. ${ }^{32}$

\section{Mucus Permeation Study}

In order to investigate the permeability of several insulinbased nanoparticles with respect to mucus, we used transwell chamber experiments. ${ }^{33}$ Firstly, we used a buffer solution with $\mathrm{pH}$ of 6.5 , which was prepared with disodium phosphate and sodium dihydrogen phosphate. We dissolved $20 \mathrm{mg}$ of gastric mucin into $1 \mathrm{~mL}$ of buffer solution to form artificially derived mucus. Insulin nanoparticles with FITC fluorescence were prepared following methods described in "Preparation of insulin nanoparticles section". Then, $100 \mu \mathrm{L}$ of artificial mucus was added to a transwell chamber, then $500 \mu \mathrm{L}$ insulin nanoparticles were added upon the mucus, and then $1.5 \mathrm{~mL}$ buffer solution of $\mathrm{pH} 6.5$ was added to another layer of the chamber. The 12-well plates were then placed in shakers, were incubated at $37^{\circ} \mathrm{C}$ and at $120 \mathrm{rpm}$ for $2 \mathrm{hrs}$. From the receptor chamber, $400 \mu \mathrm{L}$ of samples were collected in respective cuvettes. In addition, measures of permeability percentage were calculated by way ratios of fluorescence intensity of insulin nanoparticles passing through mucus layers against the original levels of insulin nanoparticles which were recorded to have been first introduced.

\section{Study on Cytotoxicity of Nanocarriers}

Measures of cytotoxicity of nanocarriers were evaluated by 3-4,5- dimethyl-2 - thiazolium -2,5- diphenyl -2-Htetrazolium bromide (MTT). ${ }^{34}$ Caco-2 cells were seeded in 96-well plates with $8 \times 10^{3}$ cells per well and were grouped according to concentration gradients of 0,50 , $100,250,500$, and $1000 \mu \mathrm{g} / \mathrm{mL}$ with 5 parallel samples. The 96-well plates were cultured in an atmosphere of 5\% carbon dioxide within an incubator set at $37^{\circ} \mathrm{C}$ for $24 \mathrm{hrs}$. Then, we added $100 \mu \mathrm{L}$ of medium per well with different drug concentrations and cells, and cultured for another $24 \mathrm{hrs}$. Next, $20 \mu \mathrm{L}$ of MTT $(5 \mathrm{mg} / \mathrm{mL})$ solution was added to each well and cells were cultured for $4 \mathrm{hrs}$. Then, liquid was carefully removed and $150 \mu \mathrm{L}$ of DMSO was added into each cell such as to allow the complete dissolution of crystals. At last, we used a microplate reader (Infinite M200 Pro, Swiss TECAN 
Company) to measure absorption values and to calculate measures related to cell viability.

\section{Cellular Uptake Study}

The distribution of drugs in cells can be observed by Confocal Laser Scanning Microscope (CLSM, FV1000, Olympus, USA). Caco-2 cells were seeded in the culture dishes at the density of $1 \times 10^{5}$ cells per well at $37^{\circ} \mathrm{C}$. The insulin NPs coated with fluorescently labeled insulin $(50 \mu \mathrm{g} / \mathrm{mL})$ or insulin were incubated with cells for $5 \mathrm{hrs}$. Then, the cells were fixed with $4 \%$ paraformaldehyde solution in dark settings for $5 \mathrm{~min}$ and stained with Hoechst33342 for $15 \mathrm{~min}$. The cells were washed with PBS three times and observed under CLSM by 20x water microscope.

Flow cytometry was used to measure the amount of FITC-insulin taken in by Caco- 2 cells, with slight modification from a previously described method. ${ }^{35}$ Caco- 2 cells were seeded with $2 \times 10^{5}$ cells per well in 6-well plates for 24 h. The cells were then incubated for $5 \mathrm{~h}$ with different nanoparticles and with transport pathway inhibitors and with final concentrations of FITC-insulin which were $100 \mathrm{mg} / \mathrm{mL}$. In detail, $10 \mu \mathrm{g} / \mathrm{mL}$ chlorpromazine, $10 \mu \mathrm{g} / \mathrm{mL}$ genistein, $10 \mu \mathrm{g} / \mathrm{mL}$ amiloride, PBS (control group) was, respectively, dissolved in the suspension of nanoparticles. ${ }^{5}$ Then, we removed the culture medium, and cells were washed with cold PBS 3 times. Next, cells were digested by using $0.25 \%$ trypsin and were then centrifuged, collected, dispersed, and finally renewed in a solution of $0.50 \mathrm{~mL}$ cold PBS for detection by use of flow cytometry (BD, ACCURI C6).

\section{Establishment of Caco-2 Cell Monolayer Model}

When cell densities reached $80 \sim 90 \%$ in the culture bottle, cells were seeded into a culture-treated polyester membrane (diameter $12 \mathrm{~mm}$, membrane area $1.12 \mathrm{~cm}^{2}$, pore size $0.4 \mu \mathrm{m}$ ) at a density of $6 \times 10^{5}$ cells $/ \mathrm{mL}$ by way of using trypsin-based digestions. Cell suspensions $(0.5 \mathrm{~mL})$ were added to the Apical (AP) chamber of the transwell culture plate and fresh complete culture medium DMEM $(1.5 \mathrm{~mL})$ was added to the basolateral (BL) chamber. On the second day, the old culture medium in transwell culture plates of the BL and AP treatment groups were discarded successively and an appropriate amount of HBSS buffer was added to wash the cell layer and the bottom of the chamber. Finally, we added $1.5 \mathrm{~mL}$ or $0.5 \mathrm{~mL}$ of fresh DMEM complete culture medium to the BL chamber and AP chambers of transwell culture plate respectively. Thereafter, the culture medium was changed daily through the 21 st day. The integrity of the model was detected by use of TEER and fluorescence yellow. ${ }^{36}$ Generally, such an approach ${ }^{37,38}$ could be used as a guideline for permeability-related experiments when TEER values are greater than $220 \Omega \cdot \mathrm{cm}^{2}$ The evaluation criterion $^{39}$ of the criteria based model assessments was as follows: apparent permeability (Papp) coefficient of fluorescence yellow was less than $1 \times 10^{-6}$.

\section{Transepithelial Transport of Insulin NPs}

Caco-2 cells were seeded upon Transwells and were cultured for 21 days as previously described. ${ }^{40}$ Old culture mediums were discarded and preheated Hank's Balanced Salt Solution (HBSS) was added to wash cell metabolites and residual medium. Next, we added the insulin nanoparticles $(0.5 \mathrm{~mL}, 0.15 \mathrm{mg} / \mathrm{mL})$ to the AP chamber and added $1.5 \mathrm{~mL}$ of preheated HBSS to the BL chamber. The $200-\mu \mathrm{L}$ solution was extracted from the BL chamber and in amounts that were equivalent to how much HASS was supplemented. These measures were determined by way of using a porcine INS ELISA kit. In addition, the values of TEER relevant to the opening of tight junctions were measured by way of using a Millicell-Electrical Resistance System (ERS-2, Millipore, USA). The apparent permeability (Papp) value represents the ability of the tested drug to cross the monolayer of Caco- 2 cells, namely the drug transport rate. The apparent permeability coefficient of insulin was calculated according to the formula as follows:

$$
\text { Papp }=\frac{Q}{A}
$$

where $\mathrm{Q}$ is the amount of drug penetration $(\mu \mathrm{g}), \mathrm{A}$ is the membrane area, which is typically a value $=1.12 \mathrm{~cm}^{2}, \mathrm{C}$ is the initial concentration $(\mu \mathrm{g} / \mathrm{mL})$ of the drug in the donor chamber, and $t$ is the total time of the experiment (s).

\section{Observation of Morphological Transformation of Tight Junctions}

To investigate changes in the tight junctions on the model monolayer of Caco- 2 cells at the predetermined time point, ${ }^{41}$ the cells were incubated with insulin nanoparticles or insulin solution for $2 \mathrm{~h}$. Thereafter, cells were incubated with $4 \%$ polyformaldehyde for 15 mins, were washed with PBS, and were then treated with anti-ZO-1 $(5 \mu \mathrm{g} / \mathrm{mL})$ at room temperature for $30 \mathrm{~min}$. Then, the cells were 
incubated with a secondary antibody labeled for fluorescence $(10 \mu \mathrm{g} / \mathrm{mL})$ for 30 mins. After washing with PBS, the cells were photographed with CLSM.

\section{In situ Intestinal Absorption Study}

The absorption of insulin NPs in intestinal villi has been previously examined in situ in an intestinal loop-based model. ${ }^{33}$ All animal experiments were carried out in compliance with the Animal Management Rules of the Ministry of Health of the People's Republic of China (document no. 55, 2001) and were approved by the Committee on Medical Ethics of the Qingdao University Affiliated Hospital (Qingdao, China). We used a rat-based model, rats were fasted for $12 \mathrm{hrs}$ prior to the experiment, and were then anesthetized with an application of $1 \%$ pentobarbital sodium solution $(70 \mathrm{mg} / \mathrm{kg})$. The abdomens of mice were opened and sections of the intestinal loop = $2 \mathrm{~cm}$ were ligated. We used fluorescently labeled insulin solutions and insulin nanoparticles $(500 \mu \mathrm{L})$ which were injected into the loop and abdomens, which were then sutured by the use of suturing needles. After administration for $1 \mathrm{hr}$, the loops were removed and the internally dissolved substances were washed with ice-cold physiological hydrochloric acid. Then, loops were fixed with $4 \%$ paraformaldehyde, which were sanctioned into $\mu \mathrm{m}$-sized slices $^{34}$ and then labeled with DAPI. Lastly, the sections were observed by way of using CLSM.

\section{Hypoglycemic Effect of Insulin Nanoparticles in vivo}

Male balb/c rats weighing $20.0 \pm 1.0 \mathrm{~g}$ were purchased from Qingdao Daren Fucheng Animal Husbandry co. Ltd. (China) and were housed as previously described. ${ }^{42}$ All animal experiments were carried out in compliance with the Animal Management Rules of the Ministry of Health of the People's Republic of China (document no. 55, 2001) and were approved by the Committee on Medical Ethics of the Qingdao University Affiliated Hospital (Qingdao, China). Experimental animals were kept under a $12 \mathrm{~h}$ light $12 \mathrm{~h}$ dark cycle, were fed with a standard laboratory rodent diet, and were provided with tap water. To induce type 1 diabetes, rats were intraperitoneally injected with $60 \mathrm{mg} / \mathrm{kg}$ streptozotocin (STZ) in sodium citrate buffer ( $\mathrm{pH} 4.2$ ) after fasting for $12 \mathrm{~h}$. Measures of the levels of fasting blood glucose of rats were tested with continuous injections of STZ across a 10-day period. Rats were judged as having hyperglycemia in our model when fasting blood glucose levels were found to have been higher than 11.1 $\mathrm{mmol} / \mathrm{L}$. In order to prepare for insulin nanoparticle administration, rats were divided into groups for: 1) subcutaneous injections of insulin solutions (positive control); 2) for orally consumed deionized water (blank control); 3) free insulin solution (negative control); and 4) HA-DCDA-CSINS NPs and HA-DCDA-CS-r8-INS NPs treatment groups. Blood samples were taken from the tail vein at 1, 2, 4, 6, 8, 10 , and $12 \mathrm{~h}$ before and post-administration of respective treatments. Measures of the levels of blood glucose were determined using sannuo glucometers and corresponding test papers at predetermined time points.

\section{Statistical Analyses}

Data were analyzed using descriptive statistics, single-factor analyses of variance (ANOVA), and were presented as mean values \pm standard deviation (SD) derived averaged across three to five independent measurements. Comparisons among treatment groups were performed by independent sample Student's $t$-tests. Differences were considered as significant and the null hypothesis of no difference among treatment groups was rejected for $\mathrm{P}$ values $<0.050$.

\section{Results and Discussion Synthesis and Characterization of DCDA-CS}

Chitosan was found to be difficult to dissolve in common solvents except for within acidic solutions with $\mathrm{pH}<6.5$. For achievement of higher water solubility of chitosan, we determined that The amino groups of chitosan reacted with quaternary carbon of dicyandiamide under the action of potassium persulfate and DCDA-CS. This observation allowed us to successfully synthesize DCDA-CS (Figure 2A). The results of water solubility analyses indicated that chitosan was only soluble in hydrochloric acid and 1\% acetic acid but that it was insoluble in water, dimethyl sulfoxide (DMSO), tetrahydrofuran, and (THF) (Table 1). However, a good result was that novel synthesized DCDA-CS was soluble in water, which illustrated that water solubility of chitosan was improved postgrafting of the dicyandiamide group.

The IR spectrum was recorded by the use of an IR-based spectrometer (Nicolet iS50, Thermo Fisher Scientific, USA) and within the range of $4000 \sim 5000 \mathrm{~cm}^{-1}$. The results indicated that characteristic peaks of sugar rings of CS were present at $1153.62,1088,1032$, and at $987.1 \mathrm{~cm}^{-1}$, whereas peaks for DCDA-CS were present at 1152.3, 1083.5, 1052.1 , and $1023.7 \mathrm{~cm}^{-1}$. The differences were likely due 


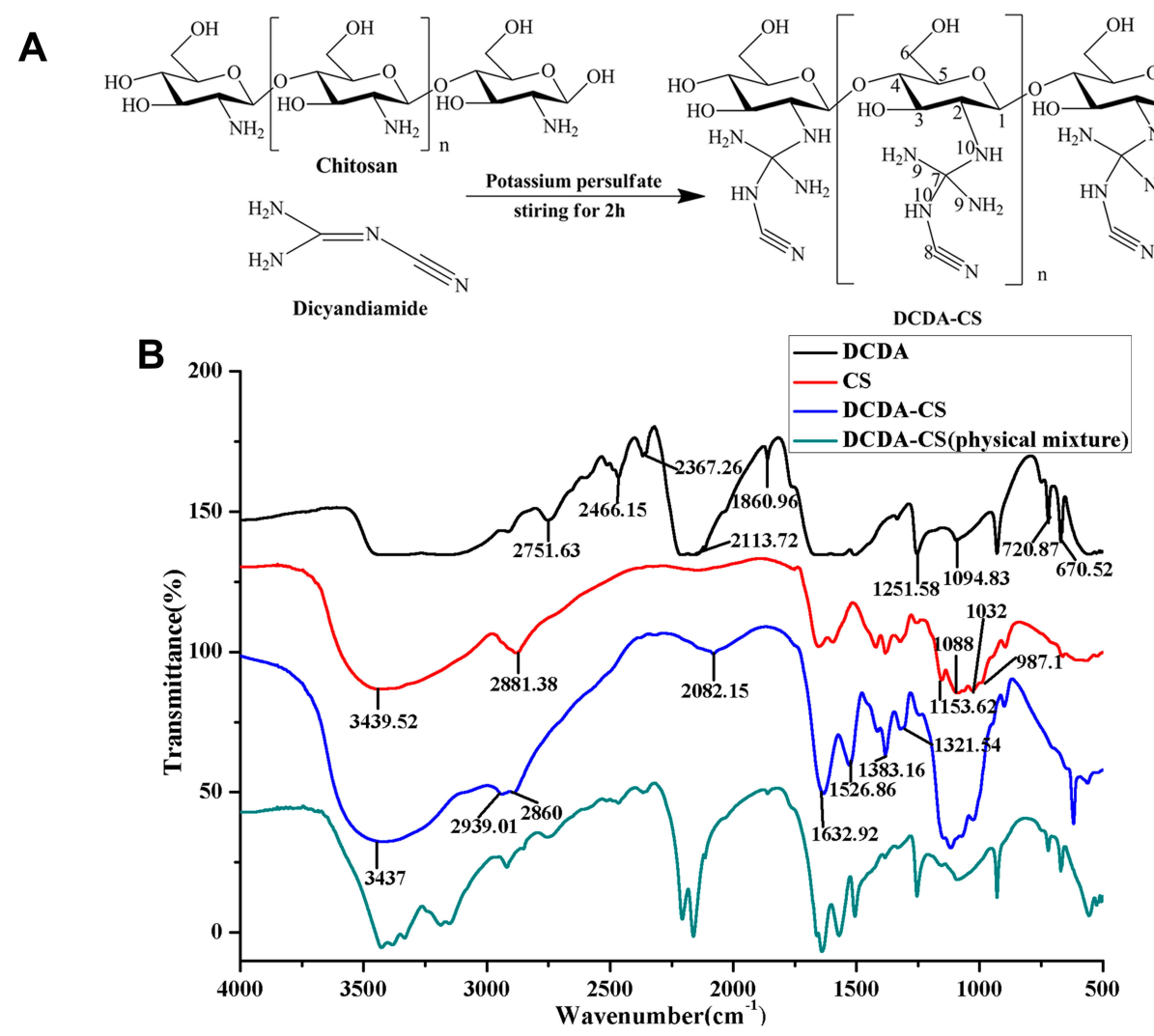

Figure 2 Synthesis and characterization of DCDA-CS. (A) Schematic routine for DCDA-CS. (B) IR spectra of DCDA (black), CS (red), DCDA-CS (blue) and physical mixture of DCDA-CS (green).

to $\mathrm{O}-\mathrm{H}$ bending, $\mathrm{C}-\mathrm{O}$ stretching, and $\mathrm{C}-\mathrm{N}$ stretching which can be seen in Figure 2B. In this figure, CS and DCDA-CS have strong peaks around $3400 \mathrm{~cm}^{-1}$ where the stretching vibration of polysaccharide bonds occurs and underlies dynamics such as related to O-H, N-H, and hydrogen stretching vibration. The infrared spectra-based results from DCDA-CS analyses indicated that the new stretching frequency of nitrile groups $(-\mathrm{CN})$ of DCDA occurred at $2082.15 \mathrm{~cm}^{-1}$, compared with that of CS. Beyond that, the $\mathrm{N}-\mathrm{H}$ vibration band of DCDA-CS was found to have occurred at $1632.92 \mathrm{~cm}^{-1}$ which confirmed the presence of primary amines $\left(-\mathrm{NH}_{2}\right)$ in DCDA-CS. The stretching bands at $2939.012860 \mathrm{~cm}^{-1}$ that were caused by $\mathrm{C}-\mathrm{H}$ were also observed. From what has been discussed above, the synthesis of DCDA-CS was confirmed by using IR-based spectral analyses and through dynamics underlying water solubility.

CS does not dissolve in water but, rather can be diluted by using inorganic or organic acids. ${ }^{21}$ And it is difficult for CS to electrostatically self-assemble with insulin in the absence of chemical crosslinkers. ${ }^{22}$ On the one hand, harsh dissolution conditions hinder the electrostatic interactions with insulin whereas. On the other hand, it is unfavorable for the stabilization of insulin when preparing insulin nanoparticles. In this study, DCDA was grafted onto chitosan to form (DCDA-CS) by using KPS as initiator at temperature up to $60^{\circ} \mathrm{C}$, the free amino group in the chitosan bonded to the double bond in dicyandiamide, resulted in triple bonds which allow further polymerization. ${ }^{21}$ The newly synthesized DCDA-CS was designed to be able to dissolve in water owing to the protonation of plentiful amino groups in polymers. Consequently, this would improve the solubility of CS and help to meet the requirements of preparing insulin under neutral conditions and with high stability.

\section{Optimization of Conditions to Prepare Uniform Insulin Nanoparticles}

The $\mathrm{pH}$ value of insulin solution and the mass ratio of DCDA-CS to insulin (INS) were systematically screened to prepare DCDA-CS-INS NPs with uniform sizes and with consistently high encapsulation efficiencies. The isoelectric point of insulin we employed was about 5.3. Insulin, when negatively charged, can form nanoparticles 
Table I Water Solubility Test of DCDA-CS

\begin{tabular}{|l|l|l|}
\hline Solvents & CS & DCDA-CS \\
\hline $\mathrm{H}_{2} \mathrm{O}$ & Insoluble & Soluble \\
$\mathrm{HCL}$ & Soluble & Soluble \\
$\mathrm{I} \%$ Acetic acid & Soluble & Soluble \\
$\mathrm{DMSO}$ & Insoluble & Insoluble \\
THF & Insoluble & Insoluble \\
Acetone & Insoluble & Insoluble \\
Isopropanol & Insoluble & Insoluble \\
\hline
\end{tabular}

with electropositive DCDA-CS by way of electrostatic self-assembly and when $\mathrm{pH}$ is greater than $5.3 .^{35}$ The minimum average particle size was $139.5 \mathrm{~nm}$ when the $\mathrm{pH}$ value of insulin solution $=7.0$ (Figure $3 \mathrm{~A}$ ). In addition, the Zeta-potential decreased gradually when the $\mathrm{pH}$ value of insulin solution was $>7.0$ because insulin becomes more electronegative, which consequently leads to a lower Zeta-potential of insulin nanoparticles. Besides, the smallest average particle size appeared when the mass ratio of DCDA-CS to insulin was 1:1 (Figure 3B). On this condition, the Zeta-potential was observed to be about 20 $\mathrm{mV}$ and this corresponded to the highest levels of both entrapment efficiencies and drug loading (Figure 3C).

In the experiment of mass ratio, the ratio of DCDA-CS to INS decreased successively with the invariant initial concentration of each component. In effect, the concentration of insulin increased gradually in the system (the mass of insulin was constant), thus it was easier for insulin to electrostatically self-assemble with DCDA-CS. In general, entrapment efficiency correspondingly increased with higher insulin incubation concentrations, ${ }^{43}$ but, there was a failure of NPs to become stable at lower mass ratios of $0.5: 1$ after the experiment. Therefore, nanoparticles were prepared with insulin and at $\mathrm{pH}=7.0$ and with a mass ratio of $1: 1$, which were conditions beneficial and that helped to obtain uniform insulin nanoparticles with high encapsulation.

\section{Preparation and Characterization of Insulin Nanoparticles}

In our experiments, DCDA-CS-INS NPs, DCDA-CS-INS-r8 -INS NPs, HA-DCDA-CS-INS NPs, and HA-DCDA-CS-r8INS NPs were prepared, and all spontaneously formed nanoparticles through electrostatic self-assembly in water. ${ }^{44}$ All mean sizes of insulin NPs were found to be less than $200 \mathrm{~nm}$, which are regarded as favorable sizes for cellular uptake (Figure 4A). ${ }^{45}$ Additionally, spherical morphology with a smooth surface and uniform dispersion of insulin NPs were demonstrated by way of assessments from morphological characterization of transmission electron microscopy (Figure 4AD).

The characterizations of NPs including size, zeta potential, polydispersity index (PDI), encapsulation efficiencies


Figure 3 Optimization of preparation conditions. (A) Mean size and Zeta-potential of DCDA-CS-INS NPs with different pH of insulin. (B) Mean size and Zeta-potential of DCDA-CS-INS NPs with different mass ratio of DCDA-CS and INS. (C) Entrapment efficiency and drug loading of DCDA-CS-INS NPs with different mass ratio of DCDACS and INS. 
DCDA-CS-r8-INS NPs

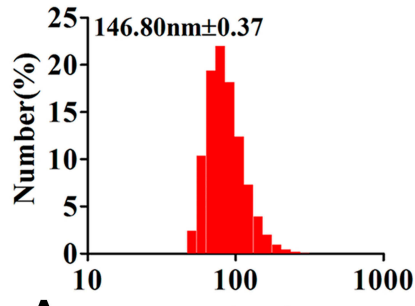

A $\quad$ Size $(\mathbf{n m})$

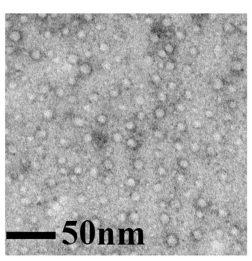

E

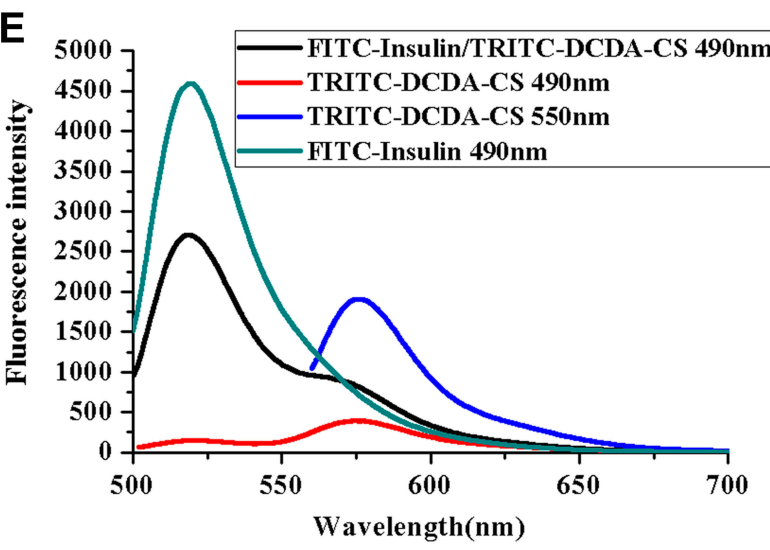

B Size $(\mathbf{n m})$

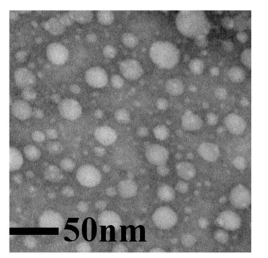

HA-DCDA-CS-r8-INS NPs

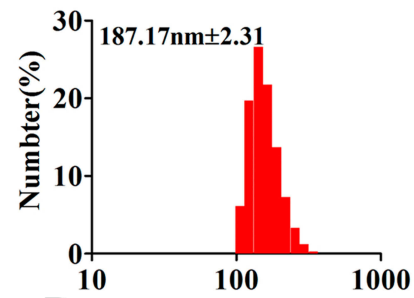

F

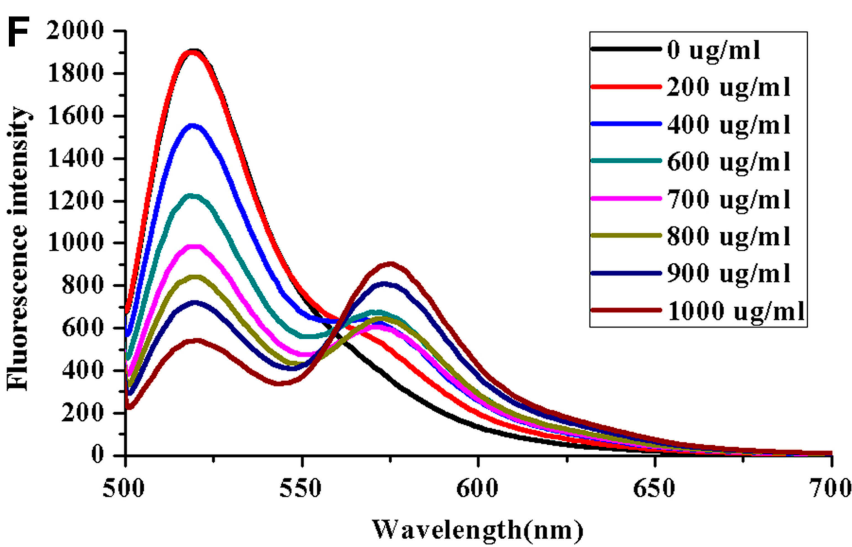

Figure 4 Characterizations of NPs. Particle size distribution and transmission electron microscope of DCDA-CS-r8-INS NPs (A), HA-DCDA-CS-r8-INS NPs (B), DCDACS-INS NPs (C), and HA-DCDA-CS-INS NPs (D). (E) Emission spectrum of NPs with FITC-labeled insulin and TRITC-labeled DCDA-CS, excited at $490 \mathrm{~nm}$ and $550 \mathrm{~nm}$. (F) Emission spectrum of NPs with different concentration of TRITC-labeled DCDA-CS, excited at $490 \mathrm{~nm}$.

(EE \%), and drug loading (DL \%) can be seen in Table 2. HA tended to be protonized in neutral water because of rich carboxyl groups, and it showed a strong negative electricity. Therefore, it could form new NPs with positively charged DCDA-CS-INS NPs or DCDA-CS-r8-INS NPs through self-assembled technology. After the addition of HA, the polydispersity of NPs was 0.039 or 0.043 , indicating that NPs had better homogeneity. Negative Zeta-potential of HADCDA-CS-INS-r8-INS NPs indicated that HA-coated DCDA-CS-r8-INS NPs successfully and that uniform and stable dispersion systems formed. In addition, there were lower measures of EE \% and DL \% of HA-DCDA-CS-INS NPs and HA-DCDA-CS-INS-r8-INS NPs. As a negatively charged macromolecule, HA could compete with insulin that also negatively charged. For example, when DNA/siRNA or other negatively charged biological macromolecules are assembled with cation materials, the addition of HA component will lead to decrease in encapsulation efficiency. ${ }^{46}$
To verify if insulin was loaded into DCDA-CS successfully, FITC and TRITC were used to label insulin and materials for DCDA-CS, respectively, and then insulin nanoparticles were prepared to form a fluorescence resonance energy transfer (FRET) pair. The emission intensity of FITC-insulin decreased at $520 \mathrm{~nm}$ and the emission intensity of TRITC increased at $573 \mathrm{~nm}$, which indicated that there was a transfer of energy from donor to acceptor when the FITC-insulin/TRITC-DCDA-CS NPs were excited at $490 \mathrm{~nm}$ (Figure 4E). In addition, the concentrations of TRITC-DCDA-CS were increased successively under treatments with the same solutions and concentrations of FITC-insulin, and when the emissions were measured at the wavelength of $490 \mathrm{~nm}$ (Figure 4F). It was found that the emission fluorescence intensity of FITCinsulin decreased gradually but for TRITC-DCDA-CS increased. Therefore, we confirmed that the fluorescence resonance energy transfer between DCDA-CS and insulin 
Table 2 Characterizations of DCDA-CS-INS NPs, DCDA-CS-INSR8-INS NPs, HA-DCDA-CS-INS NPs and HA-DCDA-CS-R8-INS NPs

\begin{tabular}{|l|l|l|l|l|l|}
\hline Sample & $\begin{array}{l}\text { Size } \\
(\mathbf{n m})\end{array}$ & PDI & $\begin{array}{l}\text { Zeta- } \\
\text { Potential } \\
(\mathbf{m V})\end{array}$ & $\begin{array}{l}\text { EE } \\
\mathbf{( \% )}\end{array}$ & $\begin{array}{l}\text { DL } \\
\mathbf{( \% )}\end{array}$ \\
\hline DCDA-CS- & 166.93 & 0.115 & $20.33 \pm 0.90$ & 75.63 & 37.87 \\
INS NPs & \pm 3.49 & \pm 0.014 & & \pm 1.09 & \pm 0.59 \\
DCDA-CS-INS & 146.80 & 0.162 & $17.63 \pm 0.53$ & 80.57 & 40.28 \\
-r8 NPs & \pm 0.37 & \pm 0.01 & & \pm 2.53 & \pm 1.55 \\
HA-DCDA-CS & 161.77 & 0.039 & $-16.93 \pm 0.41$ & 45.43 & 14.27 \\
-INS NPs & $\pm I .15$ & \pm 0.03 & & \pm 3.00 & \pm 1.09 \\
HA-DCDA-CS & 187.17 & 0.043 & $-15.33 \pm 0.45$ & 59.91 & 16.21 \\
-r8-INS NPs & \pm 2.31 & \pm 0.02 & & \pm 1.53 & \pm 1.08 \\
\hline
\end{tabular}

produced. These findings helped to support that there was formation of DCDA-CS-insulin nanoparticles.

In the preparation process, DCDA-CS has a positive charge owing to the protonation of amino groups in water. Furthermore, insulin has a negative charge at the $\mathrm{pH}$ of 7. Meanwhile, carboxyl groups abound in HA, which can be employed as an easy method for deprotonization under neutral conditions such that it is electronegative. Therefore, HA can form new NPs through electrostatic self-assembly with electropositive DCDA-CS-INS NPs or DCDA-CS-INS-r8-INS NPs. In addition, water-soluble DCDA-CS contributes to the preparation of insulin nanoparticles under neutral conditions, which is beneficial to subsequent insulin stability, in steps related to preparation, as well as for aspects related to safety.

The in vitro insulin release behavior from NPs was assessed in SGF ( $\mathrm{pH} \mathrm{2.0)}$ for the first $2 \mathrm{~h}$ and then in SIF (pH 6.8) for another $6 \mathrm{~h}$ to imitate the GI environment without enzyme. As shown in Supporting Information Figure S2, HA-DCDA-CS-r8-INS NPs exhibited sustained release profiles over time, with insulin released about $10 \%$ for the first $2 \mathrm{~h}$ in SGF and finally 58-64\% for another 6 $\mathrm{h}$ in SIF. While DCDA-CS-r8-INS NPs showed significantly faster release in SGF, with insulin released about 29-36\%. It could be speculated that HA-DCDA-CS-r8INS NPs are mainly released in intestinal fluid and rarely in gastric fluid.

\section{Stability and Mucus Permeation for Insulin Nanoparticles Stability of Preparation}

In order to verify the stability of insulin nanoparticles, secondary protein structure was determined by circular dichroism (CD) with inclusion into nanoparticle formulations compared to insulin solutions. The peaks of original levels of insulin were found at $208 \mathrm{~nm}$ and $222 \mathrm{~nm}$, which are nearby to ultraviolet regions, which are known to correspond to the $\alpha$-helix and the $\beta$-turn, respectively (Figure 5A). The spectra of nanoparticles only with DCDA-CS changed from $210 \mathrm{~nm}$ to $220 \mathrm{~nm}$, indicating that there was a slight degradation in insulin components when compared with the original levels of insulin. Characteristic peaks of HA-DCDA-CS-INS NPs and HADCDA-CS-INS-r8 NPs remained intact, which confirmed that these performed better stability than nanoparticles only coated with DCDA-CS in the preparation process. While NPs coated only with chitosan alone were beneficial in helping to maintain insulin stability, the stability of NPs was found to have been significantly improved when HA and CS were used as an additional coating on the outside of the molecules.

To perfect the characterization of insulin secondary structure, CDNN software was used for the estimation of insulin secondary structure in the far-ultraviolet region (180 260 nm). CDNN estimations were based upon comparisons between CD spectra of the test protein and reference profiles of known original insulin structures. In different types of insulin NPs, the approximate proportion of $\alpha$-helix, $\beta$-sheet, $\beta$-turn, and random coil were estimated. The $\alpha$-helix of HA-DCDA-CS-INS NPs and of HA-DCDA-CS-r8-INS NPs accounted for 33.331.1\%, respectively, with little change in values compared with the original values for original levels of insulin (Table 3 ). The proportion of HA-DCDA-CS-r8-INS NPs was the most similar to the original levels of insulin, which illustrated that HA-DCDA-CS-r8-INS NPs were the best insulin protector and which helped to support our other prior conclusions.

\section{Enzyme Stability}

Based upon determinations of the best stability of HADCDA-CS-r8-INS NPs in the preparation process, enzyme stability was further assessed by circular dichroism (CD). ${ }^{4}$ $\mathrm{CD}$ spectrums for the insulin group were significantly changed in simulations of gastric fluid (SGF) treatments and demonstrated that the secondary structure was obviously destroyed (Figure 5B and C). Compared with the insulin solution, the degradation of insulin in the nanobased preparation was almost negligent. Similarly, according to the results of CDNN evaluation, we found that there were great changes in values of insulin group such as 

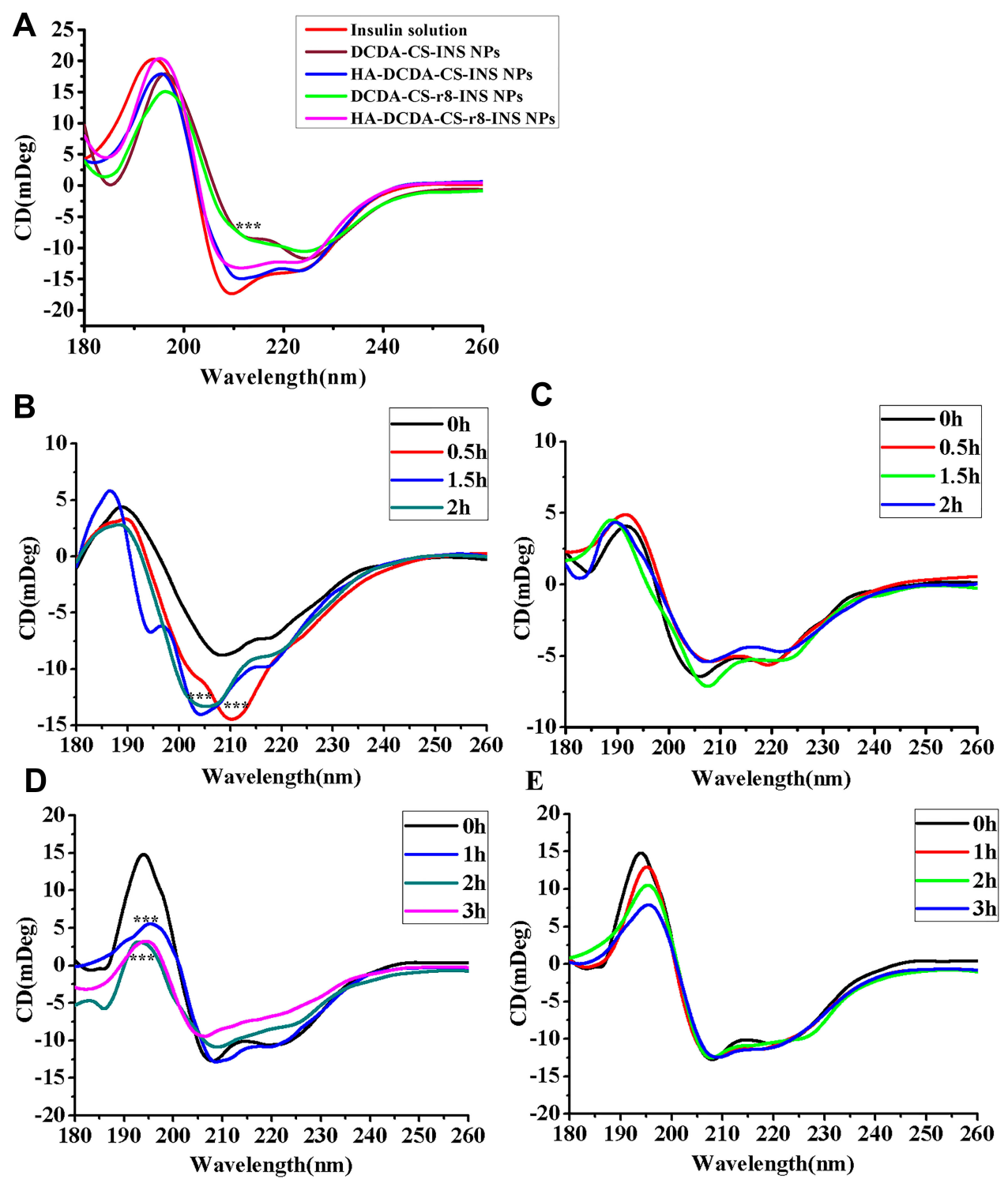

Figure 5 The stability study. (A) Circular dichroism spectra of control insulin solution and insulin preparations, $0.15 \mathrm{mg} / \mathrm{mL}$ of insulin. Proteolysis studies of insulin solution (B) and HA-DCDA-CS-r8-INS NPs (C) with simulated gastric fluid (SGF), pH=2.0. Proteolysis studies of insulin solution (D) and HA-DCDA-CS-r8-INS NPs (E) with Simulated Intestinal Fluid (SIF), $\mathrm{pH}=6.8$. $* * * \mathrm{P}<0.001$.

including for $\alpha$-helix falling from $35.1 \%$ to $25 \%$ over a 2 $\mathrm{h}$ period (Table 4). However, CDNN values of nano preparation at $2 \mathrm{hrs}$ were still not significantly different from those observed at $0 \mathrm{hr}$, which further indicated that to some extent, nano preparation coated by HA and DCDACS protected insulin from pepsin degradation.

We observed that the $\mathrm{CD}$ spectrum of the insulin group decreased dramatically at $200 \mathrm{~nm}$ after treatment with simulated intestinal fluid (SIF). This assessment also revealed that insulin was rapidly degraded (Figure 5D and E). However, the degradation rate of insulin in the nano preparation group was slower than we observed in the insulin solution group. A similar test was followed up based upon a result whereby the variation in ranges of $\alpha$ helix, $\beta$-sheet, $\beta$-turn, and random coil were determined to be about $20 \%$ in the insulin solution treatment group after treatment with simulated intestinal fluid for $3 \mathrm{~h}$. In contrast, in the nano preparation group, the range of variation was less than $4 \%$ from the analytical-based results of CDNN (Table 5). The sum of all these findings indicated that to some extent, nano preparations that included both HA and DCDA-CS served to help protect insulin from trypsin-based degradation.

In summary, the findings indicated that the HA-DCDACS-r8-INS NPs with both DCDA-CS and HA conferred stability with respect to SGF and SIF and to some extent 
Table 3 CDNN Deconvolution of the Control Insulin Solution, DCDA-CS-INS NPs, HA-DCDA-CS-INS NPs, DCDA-CS-R8INS NPs, and HA-DCDA-CS-R8-INS NPs

\begin{tabular}{|c|c|c|c|c|c|}
\hline $\begin{array}{l}\text { CDNN } \\
180- \\
260 \mathrm{~nm}\end{array}$ & $\begin{array}{l}\text { Insulin } \\
\text { Solution }\end{array}$ & $\begin{array}{l}\text { DCDA- } \\
\text { CS- } \\
\text { INS } \\
\text { NPs }\end{array}$ & $\begin{array}{l}\text { HA- } \\
\text { DCDA- } \\
\text { CS- INS } \\
\text { NPs }\end{array}$ & $\begin{array}{l}\text { DCDA- } \\
\text { CS-r8- } \\
\text { INS } \\
\text { NPs }\end{array}$ & $\begin{array}{l}\text { HA- } \\
\text { DCDA- } \\
\text { CS-r8- } \\
\text { INS NPs }\end{array}$ \\
\hline$\alpha$-helix \% & 34.3 & 25.9 & 31.1 & 25.6 & 33.3 \\
\hline$\beta$-sheet \% & 16.2 & 23.4 & 19.1 & 24.5 & 16.6 \\
\hline$\beta$-turn \% & 16.9 & 17.3 & 17.3 & 17.6 & 16.8 \\
\hline $\begin{array}{l}\text { Random } \\
\text { coil \% }\end{array}$ & 31.1 & 45.9 & 34.8 & 44 & 34.6 \\
\hline
\end{tabular}

can be implemented to avoid decomposition from pepsin and trypsin. In SGF, deprotonization of the carboxyl in HA was found to have induced the effect of hydrophilicity of HA that was weakened. Furthermore, the structure of hyaluronic acid tends to have a shrinking type of effect, making the NPs more tight and compact, and thus protecting insulin from destruction in gastric acid. Additionally, HA-DCDA-CS-r8-INS NPs, which are shielded from external positive charges, have a stronger ability to penetrate the mucus. Thus, the HA-DCDA-CS-r8-INS NPs were expected to help to maintain the biological activity of insulin by allowing it to be absorbed through the small intestine and into the blood where it can be absorbed with greater efficacy.

\section{Mucus Permeation Study}

The difficulty of diffusion in mucus is a major obstacle for oral insulin delivery. ${ }^{36}$ It has been demonstrated that the permeability of four insulin nanoparticles in mucus (Figure 6A). Insulin nanoparticles only with DCDA-CS showed very low permeability as about $94 \%$ of NPs were trapped by the mucus. For these and other types of positively charged polymers, they are not very easy for them to pass through the mucus barrier. The addition of hyaluronic acid coating can significantly increase penetration percentage to $24.6 \%$ in mucus, because of shielding from external positive charges. Therefore, HA-DCDA-CS-INS NPs and HA-DCDA-CS-r8-INS NPs have a stronger ability to penetrate the mucus, and thus may have increased applicability as options for disease treatment.

\section{Study on Cytotoxicity of Nanocarriers}

The most basic aspects of preparation-related research include verification of the innocuous nature of drug carrierbased materials. The blank NPs (HA-DCDA-CS-r8 NPs), the insulin-loaded NPs (HA-DCDA-CS-r8-INS NPs), and insulin were incubated with Caco-2 cells for $24 \mathrm{~h}$ at different concentrations whereupon measures of cell viability of Caco-2 cells were detected by MTT (Figure 6B). Cell viabilities were found to have been greater than $95.1 \%$ when the concentration of the blank NPs was less than $1000 \mu \mathrm{g} / \mathrm{mL}$, which demonstrated strikingly low levels of toxicity of nanocarriers. Cell activities for insulin-loaded NPs gradually decreased to $91.4 \%$ at concentrations of $250 \sim 1000 \mu \mathrm{g} / \mathrm{mL}$. Similarly, cell activities decreased to $87.2 \%$ with the increase of concentration in the insulin group, which suggested that decreases in cell activity of insulin-loaded NPs were caused by increases in insulin concentrations rather than by carrier materials. These results indicated that nanocarriers formed based upon hyaluronic acid, and that DCDA-CS possessed excellent cytocompatibility and great potential for in vivo based applications.

Table 4 CDNN Deconvolution of the Insulin Solution and HA-DCDA-CS-R8-INS NPs with SGF

\begin{tabular}{|l|l|l|l|l|l|l|l|l|l|}
\hline Insulin Solution CDNN 180-260nm & Oh & $\mathbf{0 . 5 h}$ & $\mathbf{I . 5 h}$ & $\mathbf{2 h}$ & Insulin NPs CDNN 180-260nm & $\mathbf{0 h}$ & $\mathbf{0 . 5 h}$ & I.5h & $\mathbf{2 h}$ \\
\hline$\alpha$-helix \% & 35.1 & 30.3 & 27.0 & 25.0 & $\alpha$-helix \% & 36.1 & 35.8 & 32.2 & 29.8 \\
$\beta$-sheet \% & 19.6 & 30.6 & 39.9 & 41.8 & $\beta$-sheet \% & 20.1 & 27.1 & 22.7 & 24.2 \\
$\beta$-turn \% & 18.7 & 20.7 & 22.1 & 21.8 & $\beta$-turn \% & 19.2 & 17.8 & 19 & 18.9 \\
Random coil \% & 19.4 & 17.9 & 17.8 & 22.0 & Random coil \% & 16.2 & 22.7 & 22 & 23.8 \\
\hline
\end{tabular}

Table 5 CDNN Deconvolution of the Insulin Solution and HA-DCDA-CS-R8-INS NPs with SIF

\begin{tabular}{|l|l|l|l|l|l|l|l|l|l|}
\hline Insulin Solution CDNN I80-260nm & Oh & Ih & 2h & 3h & Insulin NPs CDNN 180-260nm & Oh & Ih & 2h & 3h \\
\hline$\alpha$-helix \% & 43.1 & 36.5 & 28.4 & 26.8 & $\alpha$-helix \% & 43.2 & 43.2 & 43.3 & 39.1 \\
$\beta$-sheet \% & 11.4 & 16.4 & 29.3 & 32.2 & $\beta$-sheet \% & 11.1 & 11.6 & 11.6 & 13.9 \\
$\beta$-turn \% & 16.6 & 17.8 & 20.0 & 20.3 & $\beta$-turn \% & 16.3 & 16.3 & 16.4 & 17.1 \\
Random coil \% & 20.3 & 23.2 & 24.6 & 26.1 & Random coil \% & 21.2 & 21.2 & 21.7 & 22.4 \\
\hline
\end{tabular}



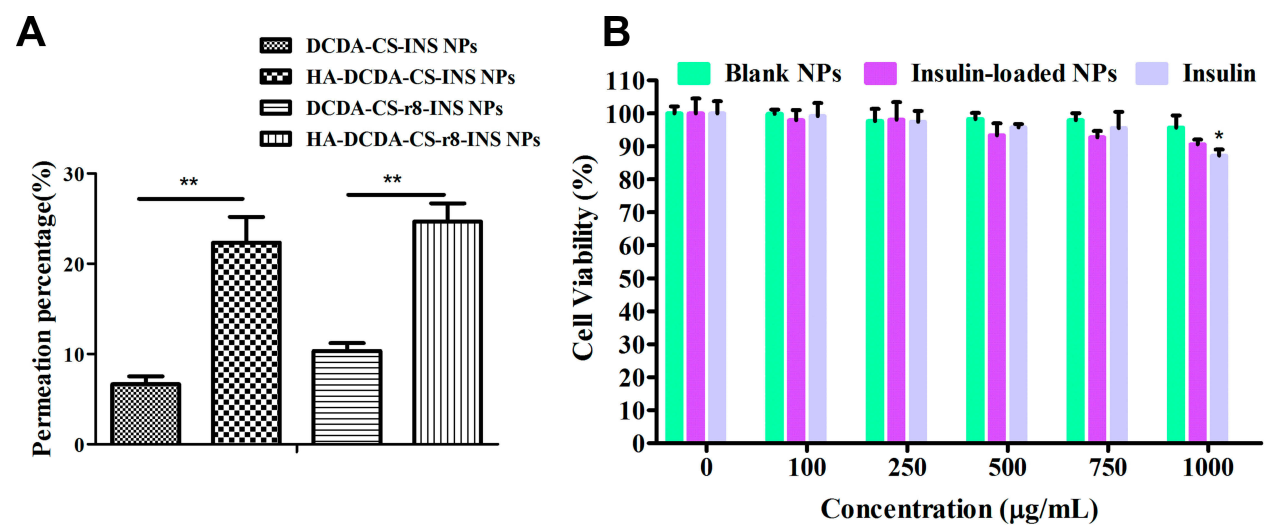

Figure 6 Mucus permeation and cytotoxicity studies. (A) Permeantion percentage of insulin NPs in artificial mucus for $2 \mathrm{~h}$. $* * \mathrm{P}<0.0 \mathrm{I}, \mathrm{N}=3$. (B) In vitro cell viability of Caco-2 cells against blank NPs (HA-DCDA-CS-r8 NPs), insulin-loaded NPs (HA-DCDA-CS-r8-INS NPs), and insulin with different concentration by MTT. *P < 0.05 vs the concentration of $0 \mu \mathrm{g} / \mathrm{mL}$. N $=5$.

\section{Studies on Cellular Uptake}

The cellular uptake of FITC-labeled insulin was determined by using flow cytometry. FITC-insulin and insulin NPs were incubated with Caco- 2 cells for equal amounts of time. The fluorescence-based intensities of NPs were successively shifted to the right compared with the group that received FITC insulin, which indicated that NPs coated CS and HA had the potential for better cellular uptake (Figure 7A). What is more, quantitative results indicated that there was a significant difference between HA-DCDA-CS-r8-INS NPs and HA-DCDA-CS-INS NPs (Figure 7B), which suggested that the HA-DCDA-CS-r8-INS NPs immensely enhance cellular uptake. Due to the presence of the cellpenetrating peptide ${ }^{47}$ and positively charged DCDA-CS, HA-DCDA-CS-r8-INS NPs had high binding rates to cells, which helped promote cellular uptake.

The confocally derived fluorescence-based microscope images showed similar results (Figure 7E). Regarding the HA-DCDA-CS-r8-INS NPs, it was observed that even after multiple washes, NPs were still present and remained in contact with the cells. The yellow color that resulted from the overlap of the green and red channels in the merged pictures revealed colocalization of DCDA-CS and insulin, which illustrated that insulin entered the cell along with nanoparticles. In contrast, HA-DCDA-CS-INS NPs showed lower measures of co-localization than were observed for HA-DCDA-CS-r8-INS NPs, which proved that the r8 variety played a significant role in promoting cellular uptake. All these findings are important because cell-penetrating peptides (CPPs) are relatively short peptides which promote cell internalization of various molecular carriers. ${ }^{28}$

To research mechanisms underlying and influencing cellular uptake, different transport inhibitors were added to each group during cellular uptake. The temperature of $4^{\circ} \mathrm{C}$ was found to have inhibited the active transport, ${ }^{35}$ and genistein was able to induce inhibition of transport pathways reliant upon the lipid raft. ${ }^{5}$ Amiloride was found to be a macropinocytosis inhibitor and chlorpromazine was a clathrin-mediated endocytosis pathway inhibitor. ${ }^{48}$ Compared with the control group, the fluorescence intensity in the $4^{\circ} \mathrm{C}$ group was the lowest and significance was identified for the genistein group, and the amiloride group (Figure 7C and D). Therefore, these results demonstrated that the active transport, lipid raft, and macropinocytosis pathways were all involved in cellular uptake of HADCDA-CS-r8-INS NPs. It has already been described that insulin was transported by active transport in previous research. ${ }^{49}$ Our findings indicated that there were no prominent differences for comparisons of uptake of FITCinsulin between the chlorpromazine group and the control group. This result suggested that cellular uptake of HADCDA-CS-r8-INS NPs was irrelevant to and did not impact the clathrin-mediated endocytosis pathway.

\section{Detection of TEER and Transepithelial Transport of Insulin}

Changes in transepithelial electrical resistance (TEER) of the Caco-2 cell monolayer were measured to determine whether or not tight junctions between cells were open. TEER values of Caco-2 cell monolayers are measures that have been widely used as indicators of permeability across the paracellular barrier. ${ }^{50}$ With the opening of tight junctions, TEER of the epithelial monolayer was found to have visibly decreased, and this was likely caused by way of transport of ions through the paracellular pathway. Since tight junction 
A

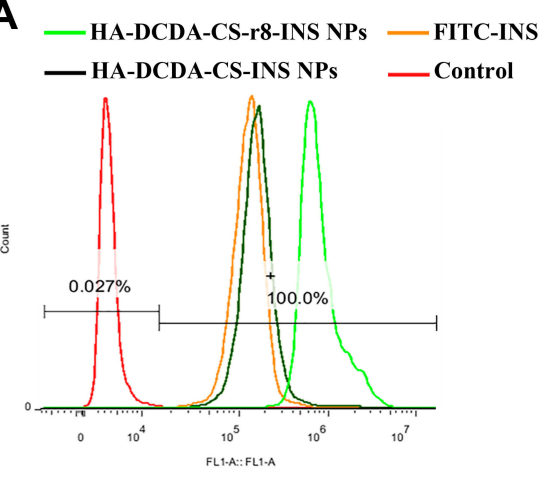

C - Chlorpromazine - Amiloride

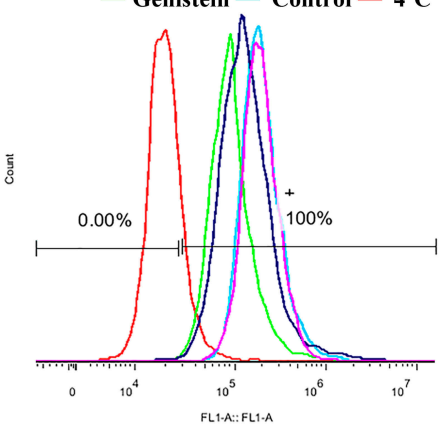

E

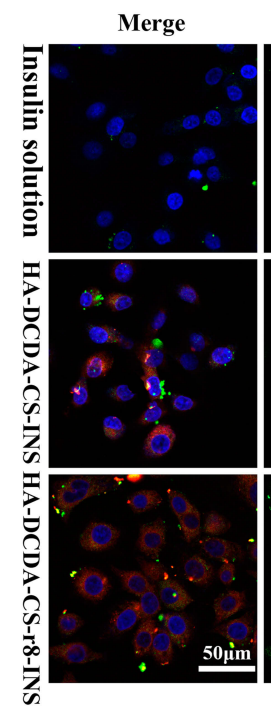

B
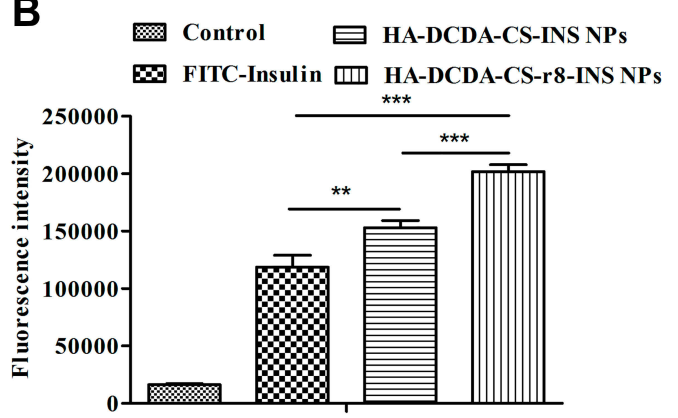

D

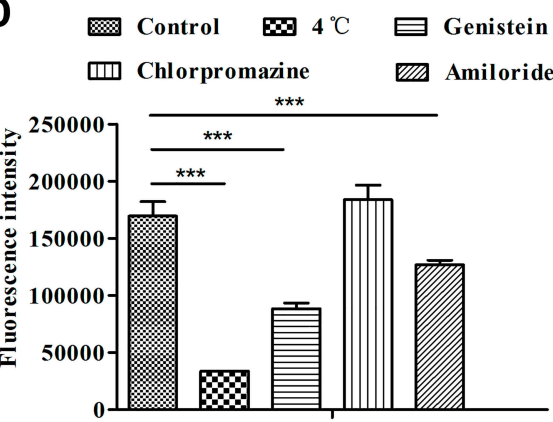

FITC-Insulin

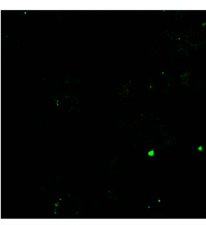

TRITC-DCDA-CS Hoechst 33342



Figure 7 Results of cellular uptake studies. (A and B) Flow cytometry quantification of the interactions of the FITC-insulin, HA-DCDA-CS-INS NPs, and HA-DCDA-CS-r8INS NPs with Caco-2 cells. (C and D) Cellular uptake of FITC-Insulin by Caco-2 cells post-incubation with HA-DCDA-CS-r8-INS NPs under different conditions. (E) Qualitative cell-NPs localization by confocal fluorescence microscopy after incubation of the NPs with the cells for $5 \mathrm{~h}$ at $37^{\circ} \mathrm{C}$. Red: DCDA-CS stained with TRITC; green: FITC-insulin; blue: cell nucleus stained with Hoechst33342; yellow: colocalization. $\mathrm{N}=3$, ***P $<0.00 \mathrm{I}$, **P $<0.0 \mathrm{I}$.

proteins are dependent on $\mathrm{Ca},{ }^{2+}$ the junctions loosen with depletion of $\mathrm{Ca}^{2+}$ concentrations. ${ }^{25}$ It has accordingly been reported that chitosan-based nanoparticles could reversibly open tight junctions. ${ }^{51}$ HA-DCDA-CS-r8-INS NPs and HADCDA-CS-INS NPs were incubated with Caco-2 cell monolayer for $2 \mathrm{~h}$, resulting in a faster decline of TEER to $65 \%$ of the initial value, which was significant in comparison with the group treated with insulin solution (Figure 8A). In addition, the recovery of TEER values was observed in both groups treated with insulin nanoparticles when samples were removed. However, there were no significant differences identified with respect to TEER values of HA-DCDACS-r8-INS NPs and HA-DCDA-CS-INS NPs possibly due to the similar content of DCDA-CS.

To further investigate the effect of insulin nanoparticles on the opening of tight junctions, morphological changes 




C
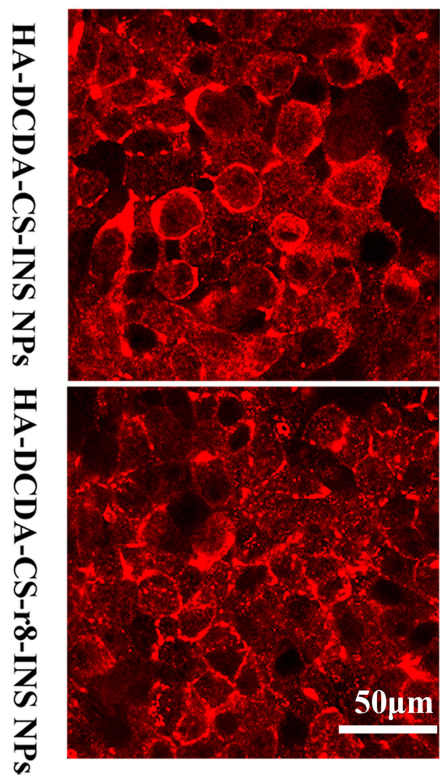

$2 \mathrm{~h}$
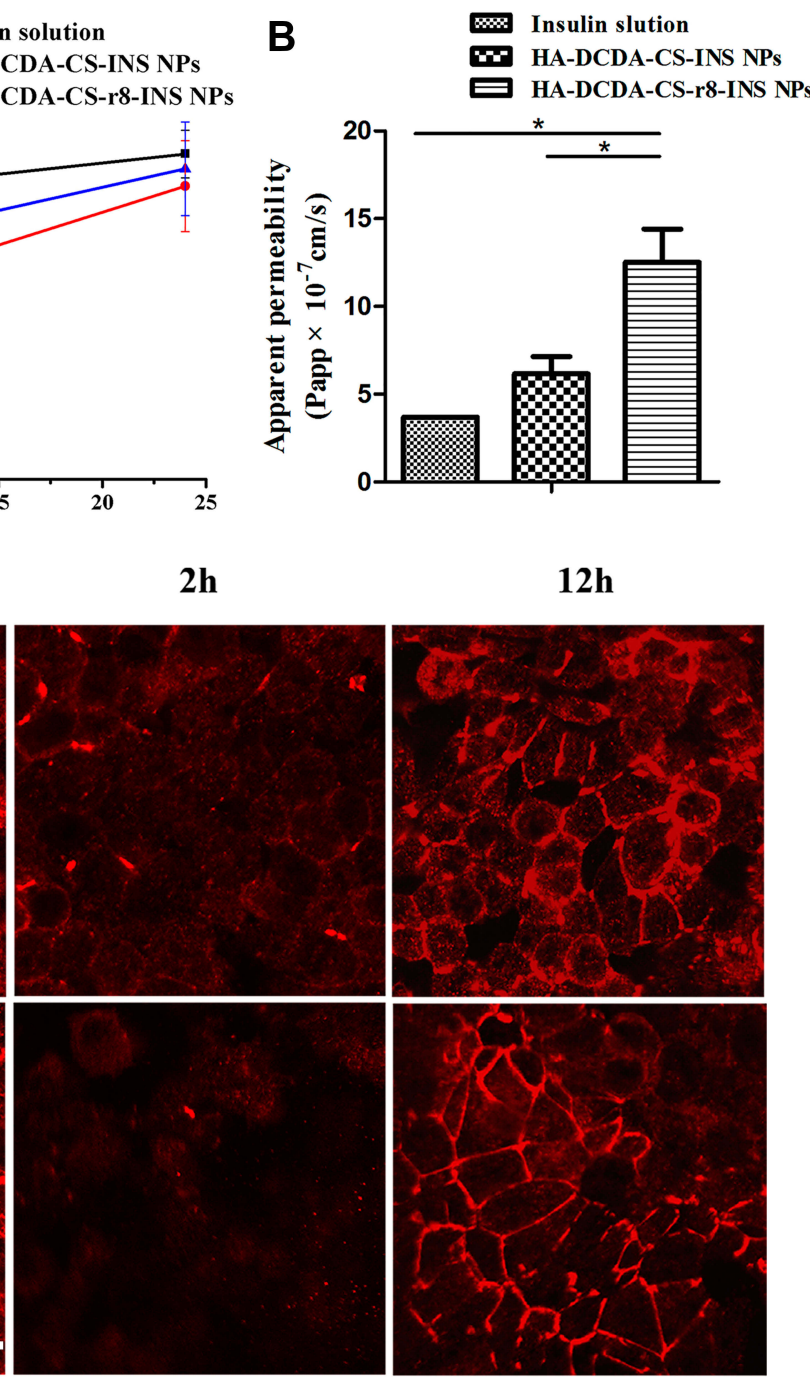

$12 \mathrm{~h}$
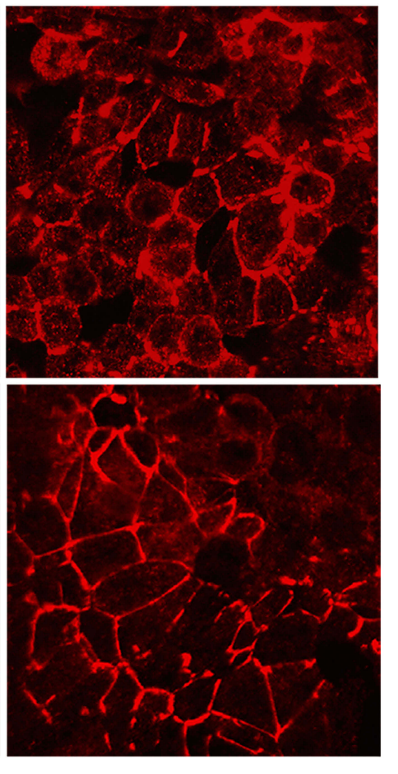

Figure 8 Transepithelial transport of insulin. (A) Effects of insulin solution, HA-DCDA-CS-r8-INS NPs, HA-DCDA-CS-INS NPs on transepithelial transport of Caco-2 cell monolayer. ${ }^{* * *} \mathrm{P}<0.00 \mathrm{I}$ vs Insulin solution group. (B) Apparent permeability coefficient (Papp) of insulin across Caco- 2 monolayer with incubation for $2 \mathrm{~h}$. $* \mathrm{P}<0.05$. (C) Fluorescence images of Caco-2 monolayer stained for tight junction protein zonula occludens-I (Zo-I) after incubation with insulin-loaded nanoparticles at different time points.

of tight junctions between Caco-2 cell monolayer incubations with insulin nanoparticles were observed by using confocal-based laser scanning microscopy (CLSM) (Figure 8C). Prior to incubation with insulin nanoparticles, the occludin formed a continuous loop around the cells. After incubation for $2 \mathrm{~h}$, expression of occludin proteins was significantly weakened and discontinuous, especially with respect to HA-DCDA-CS-r8-INS NPs, which indicated that tight junctions were opened at that time. After $2 \mathrm{~h}$, NPs were removed and the occludin gradually reappeared. Hence, thus results helped to support that insulin nanoparticles could reversibly open tight junctions, which was a finding that is also consistent with results of TEER.
The apparent permeability (Papp) value represented the ability of the tested drug to cross the monolayer of Caco-2 cells. The apparent permeability coefficient of HA-DCDACS-r8-INS NPs was $1.305 \times 10^{-6} \mathrm{~cm} / \mathrm{s}$, which was 3.56 times higher than that of insulin solution and which was 2.12 times higher than that of HA-DCDA-CS-INS NPS respectively (Figure $8 \mathrm{~B}$ ). These findings indicated that insulin nanoparticles made up of HA, DCDA-CS significantly enhanced the transmembrane transport efficiency of insulin, especially with the addition of r8. These findings also helped provide support to the idea that HA-DCDA-CS -r8-INS NPs can immensely enhance cellular uptake (Figure 7B). These effects may ultimately lead to the 
increase of apical endocytosis which may facilitate improvement in insulin transmembrane transport.

Most biological macromolecules play a therapeutic role in circulation, and likewise to these molecules, NPs are required to go through intestinal epithelial cells. ${ }^{52}$ Based upon comprehensive analyses of TEER measurements, insulin transport, and of morphological changes of the tight junctions, we found that HA-DCDA-CS-r8-INS NPs had a 3.56 times higher transmembrane apparent permeability coefficient compared with insulin solution and opened the tight junctions between Caco- 2 cells transiently and reversibly. It has been reported in the literature that the nanoparticles based on CS could mediate the transient opening of the tight junctions between enterocytes thereby preventing insulin from being kidnapped by lysosomes in the cells. ${ }^{53}$ The CS modified by DCDA in our study still had the ability to open tight junctions. In conclusion, the significant improvement in permeability could be attributed to the vital role of cell-penetrating peptides and the effect of DCDA-CS-mediated paracellular transport.

\section{In situ Intestinal Absorption Study}

To research measures of behavior of absorption of NPs in vivo, we treated the living small intestine of T1D diabetic rats with fluorescently labeled NPs or FITC-insulin ${ }^{25}$ (Figure 9A). Thereafter, the obtained isolated intestinal villi slices were visualized by using confocal laser scanning microscopy (CLSM). HA-DCDA-CS-r8-INS NPs (bottom, Figure 9A) show the highest green and red fluorescence signals in the outer edges and interiors of intestinal villi. This finding indicated that there was salient permeability of NPs into intestinal villi epithelium and then into circulating blood for oral delivered instances. HA-DCDA-CS-INS NPs (middle), by contrast, showed lower measures of fluorescence of signals but results were stronger than the insulin group (top), which confirmed HA-DCDA-CS-r8-INS NPs can enhance intestinal absorption of insulin. In particular, insulin nanoparticles self-assembled by r8, HA, and DCDA$\mathrm{CS}$ possessed the best measures as related to permeability into intestinal epithelial cells. Beyond that, the fluorescence indicated that there was co-localization of red and green, and these areas appeared yellow in color. This overlapping indicated that the insulin was carried into intestinal epithelial cells by way of nanocarriers as scheduled and predicted. It was clear that HA-DCDA-CS-r8-INS NPs were concentrated in the apical side and that NPs (yellow fluorescence) were gradually transported from apical to outer longitudinal muscle tissues (Figure 9B). These findings demonstrate the excellent ability of transcellular transport unique to HADCDA-CS-r8-INS NPs and suggested there was potential for protein drug delivery in vivo.

\section{Hypoglycemic Effect of Insulin Nanoparticles in vivo}

The diabetic rat model was established by applying intraperitoneal injections of streptozotocin (STZ) for 10 days. Fasting blood glucose levels and body weights were used as measures in evaluation-based indexes. The weight of rats in the STZ group was gradually reduced while those in the normal group gradually increased, which fit with expectations and findings for the characteristics of diabetic rats who drank more and consumed more food but still wasted away (Figure 10A). Fasting blood glucose of rats in mornings was detected by using a glucometer and blood glucose levels in T1D rats were found to have gradually increased within 10 days. After becoming drug-free, blood glucose levels stabilized and were normally characterized as larger than $11.1 \mathrm{mmol} / \mathrm{L}$ (Figure 10B), which indicated that diabetic rat model had been built successfully.

To compare the hypoglycemic effect of HA-DCDA-CS-r8 -INS NPs and HA-DCDA-CS-INS NPs in diabetic rats, equal doses of insulin were given to rats orally. In Figure 10C, oral administration of deionized water (blank control) and free insulin, solution (negative control) failed to reduce the blood glucose level, showing poor oral absorption. Subcutaneous injections of insulin solution (positive control) were performed for a normal hypoglycemic function as determined for prescheduling. An exciting finding was that oral administration of HA-DCDA-CS-r8-INS NPs gradually reduced levels to $38 \%$ of initial blood glucose within $6 \mathrm{~h}$ and that oral administration of HA-DCDA-CS-INS NPs caused a weaker decrease in blood glucose. Therefore, HA-DCDA-CS-r8-INS NPs played key roles as effective inducers of hypoglycemia, and as components that degraded slower and lasted longer when compared with subcutaneous injections of insulin.

In addition, low, medium, and high doses were administered and were designed to further study the connection between the hypoglycemic effect of insulin NPs and the applied dose (Figure 10D). In the groups which received oral doses of $30 \mathrm{IU} / \mathrm{kg}, 60 \mathrm{IU} / \mathrm{kg}$, and $120 \mathrm{IU} / \mathrm{kg}$, blood glucose levels were found to have decreased gradually from $80 \%$ to $37.8 \%$ and $27.58 \%$ of the initial value within 6 hrs. Thus, our findings lend strong support to the idea that orally administered HA-DCDA-CS-r8-INS NPs had 


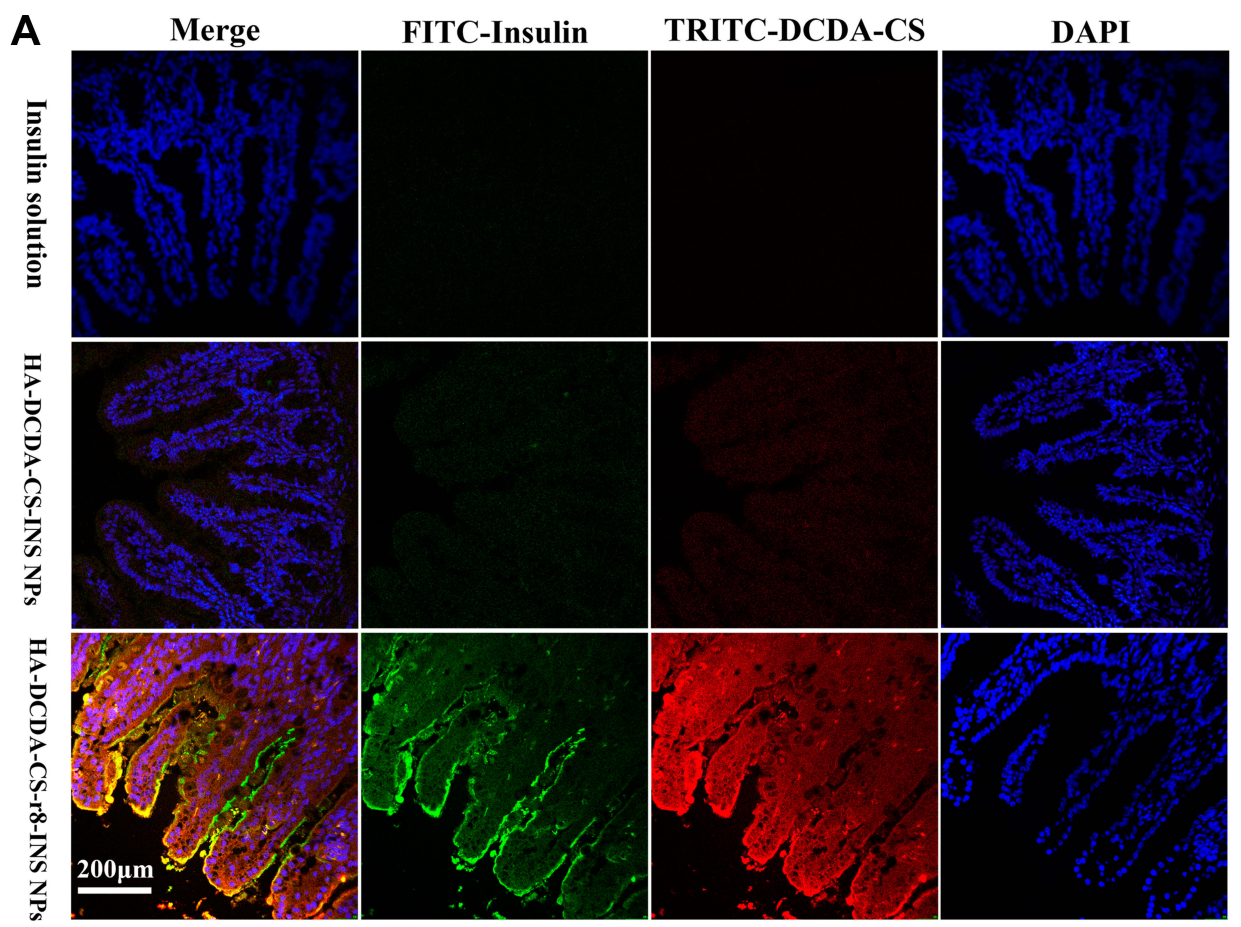

B

AP

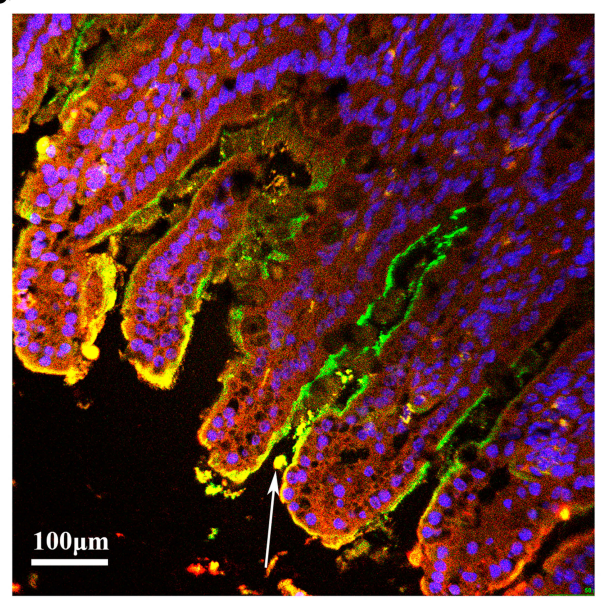

BL

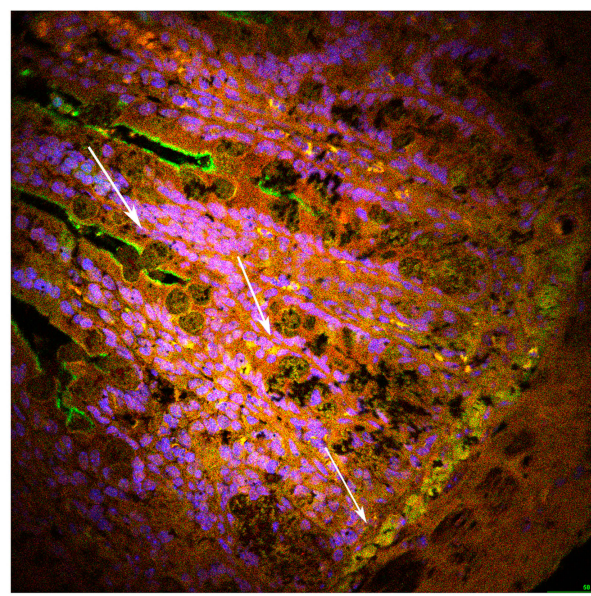

Figure 9 Intestinal sections in vitro. (A). Representative fluorescence-based images of intestinal villi after administration of fluorescently-labeled NPs in TID rats. Insulin was labeled with FITC, DCDA-CS was labeled with TRITC, and the nucleus was stained with DAPI. (B). White arrows indicate where HA-DCDA-CS-r8-INS NPs have been absorbed into the intestinal villi (yellow fluorescence).

an obvious dose-dependent hypoglycemic effect through our assessments of T1D rats.

To study measures of hypoglycemic effects of oral HA-DCDA-CS-r8-INS NPs with meals, diabetic rats were fed immediately after the administration of treatments (Figure 10E). In the group which received orally administered insulin solutions and deionized water, blood glucose levels continued to rise rapidly to about $200 \%$ of initial values. Compared with the former two groups, the HA-DCDA-CS-r8-INS NPs treatment group had significant inhibition of the rapid rise of blood glucose levels and presented a dose-dependent effect, which helped to support experimental results.

According to pharmacodynamic experimental data, oral insulin solution applications had no hypoglycemic effects whereas HA-DCDA-CS-r8-INS NPs and HA-DCDA-CSINS NPs both had obvious hypoglycemic effects, even when the relative dosages of insulin were the same. After oral administration, HA-DCDA-CS-r8-INS NPs distinctly reduced the levels of blood glucose in T1D rats and it was obvious that this was dose-dependent. Because insulin coated with hyaluronic acid and DCDA-CS is well 


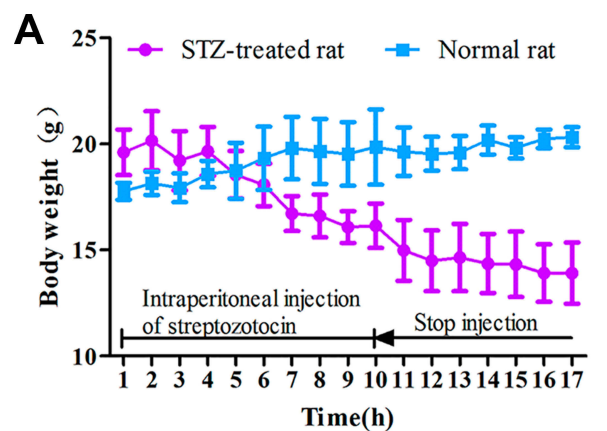

C

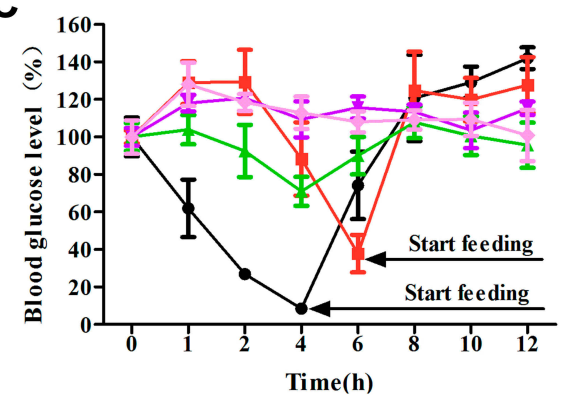

B

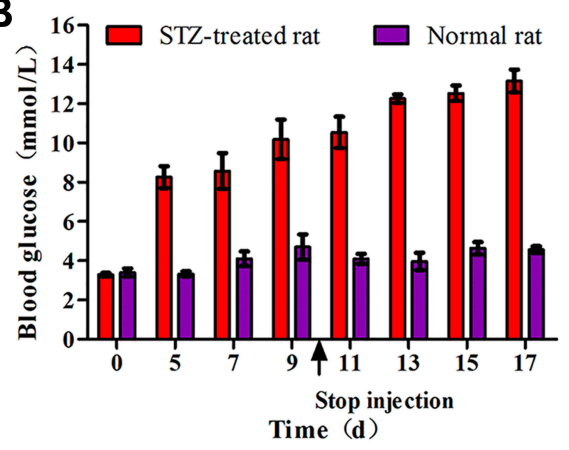

$\rightarrow$ Insulin solution (5IU/kg, s.c.)

$\rightarrow$ Deionized water (oral)

$\neg-$ Insulin solution(60IU/kg, oral)

$\dashv$ HA-DCDA-CS-INS-NPs (60IU/kg,oral)

-늘 HA-DCDA-CS-r8-INS-NPs (60IU/kg, oral)
D

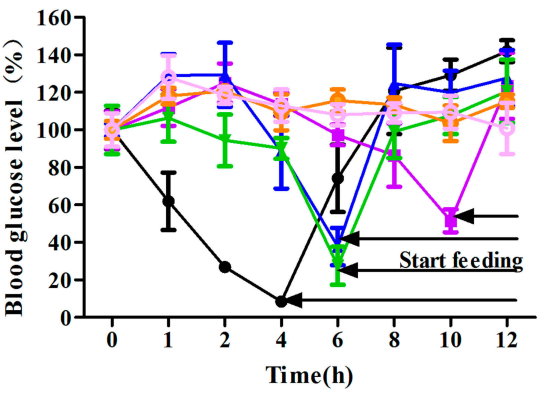

E

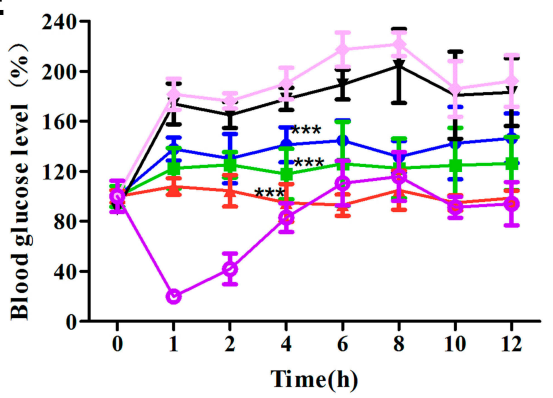

$\rightarrow$ Insulin solution $(5 I U / \mathrm{kg}$, s.c.)

- HA-DCDA-CS-r8-INS-NPs (30IU/kg, oral)

- HA-DCDA-CS-r8-INS-NPs (60IU/kg, oral)

$\rightarrow$ HA-DCDA-CS-r8-INS-NPs (120IU/kg, oral)

-O- Deionized water (oral)

$\ominus$ - Insulin solution (60IU/kg, oral)

Figure 10 Oral delivery of insulin nanoparticles on blood glucose levels in Type I diabetic (TID) rats. (A and B) Daily changes of body weight and fasting blood glucose levels after intraperitoneal injections of streptozotocin (STZ). (C) Blood glucose level in TID rats, following the administration of different insulin formulations. TID rats were fasted for $2 \mathrm{~h}$ and remained fasted during experiments with free access to water. However, the group of insulin solution and HA-DCDA-CS-r8-INS NPs started feeding at $46 \mathrm{~h}$ because of near hypoglycemia. (D) Blood glucose levels in TID rats, following the administration of different doses of HA-DCDA-CS-r8-INS NPs. TID rats were fasted for $2 \mathrm{~h}$ and remained fasted during experiments with free access to water. However, the group of insulin solution and HA-DCDA-CS-r8-INS NPs started feeding at 4 $\mathrm{h}, 6 \mathrm{~h}$, and $10 \mathrm{~h}$ because of near hypoglycemia. (E) Blood glucose levels in TID rats, following the administration of different doses of insulin formulation. TID rats were fasted for a night and were fed during these experiments. $N=6$. $* * * P<0.00 I$ vs insulin solution group.

protected from digestion and degradation in the extreme conditions of the gastrointestinal environment, this approach can beneficially increase the stability of insulin applied through oral administration. The surface of HADCDA-CS-r8-INS NPs we prepared had certain hydrophilic tendencies, which were conducive to overcoming obstacles in, and for the forms of mucus. Meanwhile, relatively properly sized insulin nanoparticles and the mucosal related adhesion properties of chitosan and HA both facilitated increases in residence time of nanoparticles in the intestinal tract. ${ }^{54}$ In addition, octaarginine (r8), as an excellent cell-penetrating peptide, promotes the entry of insulin 
NPs into cells, and then NPs slowly release insulin into circulating blood. ${ }^{28}$ What is more, NPs were involved in caveolae-mediated transport and DCDA-CS-mediated the dynamics of the paracellular pathway, which contributed to sequential transportation from the apical to basolateral areas. Taken together, HA-DCDA-CS-r8-INS NPs showed excellent in vivo hypoglycemic effects, and exhibited good correlations with in vitro studies.

\section{Conclusions}

In this study, we developed and examined a novel multifunctional oral delivery system for insulin facilitated by HA-DCDA-CS-r8-INS NPs created by way of HA and octaarginine and though electrostatic self-assembly technologies based upon DCDA-CS-INS NPs. Water-soluble DCDA-CS successfully alleviated the restrictions of the preparation condition and HA interacted with DCDA-CS, both of which made great contributions to the protection of insulin and its stability in vitro. Furthermore, HA-DCDACS-r8-INS NPs significantly increased cellar uptake through lipid raft and macropinocytosis transport and achieved transepithelial transport after oral delivery. Our results helped to confirm that the modification of r8 on NPs could significantly enhance cell permeability and efficiency of trans-epithelial cell transport, and could mediate the hypoglycemic effect. In addition, caveolae-mediated transport and reversible induction of opening of tight junctions by way of DCDA-CS could help facilitate better transmembrane transport of insulin. Furthermore, our strategy for oral administration of HA-DCDA-CS-r8-INS NPs was found to have had a strong hypoglycemic effect on T1D rats. Therefore, this multi-functional and novel-type of oral delivery system of insulin appears to have made orally applied consumption of insulin feasible and possesses potential value for wide-ranging applications.

\section{Acknowledgments}

This work was supported by the National Natural Science Foundation of China [81673360], Major Science and Technology Innovation Projects of Shandong Province [2018CXGC1408], and Science and Technology Projects for People's Livelihood of Qingdao [18-6-1-93-nsh].

\section{Disclosure}

Guizhou Hao and Guimin Zhang were employees of the Lunan Pharmaceutical Corporation during the study. The authors have declared that no other possible conflicts of interest exist.

\section{References}

1. Maher S, Mrsny RJ, Brayden DJ. Intestinal permeation enhancers for oral peptide delivery. Adv Drug Deliv Rev. 2016;106(Pt B):277-319. doi:10.1016/j.addr.2016.06.005

2. Malhaire H, Gimel JC, Roger E, et al. How to design the surface of peptide-loaded nanoparticles for efficient oral bioavailability? Adv Drug Deliv Rev. 2016;106(Pt B):320-336. doi:10.1016/j.addr.2016.03.011

3. Sosnik A, Augustine R. Challenges in oral drug delivery of antiretrovirals and the innovative strategies to overcome them. Adv Drug Deliv Rev. 2016;103:105-120. doi:10.1016/j.addr.2015.12.022

4. Presas E, McCartney F, Sultan E, et al. Physicochemical, pharmacokinetic and pharmacodynamic analyses of amphiphilic cyclodextrin-based nanoparticles designed to enhance intestinal delivery of insulin. $J$ Controlled Release. 2018;286:402-414. doi:10.1016/j.jconrel.2018.07.045

5. Sheng J, Han L, Qin J, et al. N-trimethyl chitosan chloride-coated PLGA nanoparticles overcoming multiple barriers to oral insulin absorption. ACS Appl Mater Interfaces. 2015;7(28):30-41. doi:10.1021/acsami.5b0 3555

6. Brayden DJ, Alonso MJ. Oral delivery of peptides: opportunities and issues for translation. Adv Drug Deliv Rev. 2016;106(Pt B):193-195. doi:10.1016/j.addr.2016.10.005

7. Dhuria SV, Hanson LR, Frey WH, et al. Intranasal delivery to the central nervous system: mechanisms and experimental considerations. J Pharm Sci. 2010;99.

8. Laffleur F, Hintzen F, Shahnaz G, et al. Development and in vitro evaluation of slippery nanoparticles for enhanced diffusion through native mucus. Nanomedicine. 2014;9(3):387-396. doi:10.2217/ nnm. 13.26

9. Li X, Qi J, Xie Y, et al. Nanoemulsions coated with alginate/chitosan as oral insulin delivery systems: preparation, characterization, and hypoglycemic effect in rats. Int $J$ Nanomed. 2013;8:23-32. doi:10.2147/IJN. S38507

10. Niu Z, Conejos-Sánchez I, Griffin B, et al. Lipid-based nanocarriers for oral peptide delivery. Adv Drug Deliv Rev. 2016;106(Pt B):337-354. doi:10.1016/j.addr.2016.04.001

11. Yu M, Yang Y;, Zhu C, et al. Advances in the transepithelial transport of nanoparticles. Drug Discov Today. 2016;21(7):1155-1161. doi:10.1016/j.drudis.2016.05.007

12. Ensign LM, Schneider C, Suk JS, et al. Mucus penetrating nanoparticles: biophysical tool and method of drug and gene delivery. $A d v$ Mater. 2012;24(28):87-94. doi:10.1002/adma.201201800

13. Li L, Jiang GH, Yu WJ, et al. Preparation of chitosan-based multifunctional nanocarriers overcoming multiple barriers for oral delivery of insulin. Mater Sci Eng C. 2017;70:278-286. doi:10.1016/j. msec.2016.08.083

14. Banerjee A, Ibsen K; Brown T, et al. Ionic liquids for oral insulin delivery. Proc Nat Acad Sci. 2018;115(28):7296-7301. doi:10.1073/ pnas. 1722338115

15. Fan WW, Xia DN, Zhu QL, et al. Functional nanoparticles exploit the bile acid pathway to overcome multiple barriers of the intestinal epithelium for oral insulin delivery. Biomaterials. 2018;151:13-23. doi:10.1016/j.biomaterials.2017.10.022

16. Sultankulov B, Berillo D, Sultankulova K, et al. Progress in the development of chitosan-based biomaterials for tissue engineering and regenerative medicine. Biomolecules. 2019;9(9):470. doi:10.33 90/biom 9090470

17. Liou JS, Liu BR, Martin AL, et al. Protein transduction in human cells is enhanced by cell-penetrating peptides fused with an endosomolytic HA2 sequence. Peptides. 2012;37(2):273-284. doi:10.1016/j. peptides.2012.07.019

18. Deng J, Zhang Z, Liu C, et al. The studies of N-Octyl-N-ArginineChitosan coated liposome as an oral delivery system of Cyclosporine A. J Pharm Pharmacol. 2015;67(10):1363-1370. doi:10.1111/jphp.12448 
19. Chen $\mathrm{CH}$, Lin YS, Wu SY, et al. Mutlifunctional nanoparticles prepared from arginine-modified chitosan and thiolated fucoidan for oral delivery of hydrophobic and hydrophilic drugs. Carbohydr Polym. 2018;193:163-172. doi:10.1016/j.carbpol.2018.03.080

20. Ali RM, Zeenat S, Muhammad A, et al. Self-assembled nanoparticles based on amphiphilic chitosan derivative and arginine for oral curcumin delivery. Int J Nanomed. 2016;11:4397-4412. doi:10.2147/IJN.S106116

21. Khalil ES, Saad B, Negim E-SM, et al. Novel water-soluble chitosan derivative prepared by graft polymerization of dicyandiamide: synthesis, characterisation, and its antibacterial property. J Polymer Res. 2015;22(6):116. doi:10.1007/s10965-015-0756-9

22. Sarmento B, Ribeiro A, Veiga F, et al. Alginate/chitosan nanoparticles are effective for oral insulin delivery. Pharm Res. 2007;24 (12):198-206. doi:10.1007/s11095-007-9367-4

23. Garrido AA, Molina-Bolvar JA, Galvez-Ruiz MJ, et al. Mucoadhesive properties of liquid lipid nanocapsules enhanced by hyaluronic acid. J Mol Liq. 2019;302-317.

24. Liu M, Zhang J, Zhu X, et al. Efficient mucus permeation and tight junction opening by dissociable "mucus-inert" agent coated trimethyl chitosan nanoparticles for oral insulin delivery. J Controlled Release. 2016;222:67-77. doi:10.1016/j.jconrel.2015.12.008

25. Angela MW, Margaret PG, Nicholas AP. Designing the new generation of intelligent biocompatible carriers for protein and peptide delivery. Acta Pharm Sin B. 2018;8(2):147-164. doi:10.1016/j.apsb.2018.01.013

26. Lai SK, Wang YY, Hanes J. Mucus-penetrating nanoparticles for drug and gene delivery to mucosal tissues. Adv Drug Deliv Rev. 2009;61(2):158-171. doi:10.1016/j.addr.2008.11.002

27. Khafagy El S, Morishita M. Oral biodrug delivery using cell-penetrating peptide. Adv Drug Deliv Rev. 2012;64(6):531-539. doi:10.1016/j.addr.2011.12.014

28. Rehmani S, Dixon JE. Oral delivery of anti-diabetes therapeutics using cell penetrating and transcytosing peptide strategies. Peptides. 2018;100:24-35. doi:10.1016/j.peptides.2017.12.014

29. Xu B, Jiang G, Yu W, et al. Preparation of poly (lactic-co-glycolic acid) and chitosan composite nanocarriers via electrostatic self assembly for oral delivery of insulin. Mater Sci Eng C Mater Biol Appl. 2017;78:420-428. doi:10.1016/j.msec.2017.04.113

30. Shan S, Yao J, Xu L, et al. Preparation of Icaritin-loaded mPEG-PLA micelles and evaluation on ischemic brain injury. $J$ Biomed Nanotechnol. 2019;15(4):674-685. doi:10.1166/jbn.2019.2721

31. Shan W, Zhu X, Liu M, et al. Overcoming the diffusion barrier of mucus and absorption barrier of epithelium by self-assembled nanoparticles for oral delivery of insulin. ACS Nano. 2015;9(3):45-56. doi:10.1021/acsnano.5b00028

32. Greenfield NJ. Using circular dichroism spectra to estimate protein secondary structure. Nat Protoc. 2006;1(6):2876-2890. doi:10.1038/ nprot.2006.202

33. Liu J, Cao J, Cao J, et al. Mechanistic insight into the interaction of gastrointestinal mucus with oral diblock copolymers synthesized via ATRP method. Int J Nanomed. 2018;13:2839-2856. doi:10.2147/IJN. S160651

34. Ge R, Cao J, Chi J, et al. NIR-guided dendritic nanoplatform for improving antitumor efficacy by combining chemo-phototherapy. Int J Nanomed. 2019;14:4931-4947. doi:10.2147/IJN.S203171

35. Lopes M, Shrestha N, Correia A, et al. Dual chitosan/albumin-coated alginate/dextran sulfate nanoparticles for enhanced oral delivery of insulin. J Controlled Release. 2016;232:29-41. doi:10.1016/j.jconrel. 2016.04.012

36. Yao B, He J, Yin X, et al. The protective effect of lithocholic acid on the intestinal epithelial barrier is mediated by the vitamin $\mathrm{D}$ receptor via a SIRT1/Nrf2 and NF-kappaB dependent mechanism in Caco-2 cells. Toxicol Lett. 2019;316:109-118. doi:10.1016/j.toxlet.2019.08.024

37. Ranaldi G, Consalvo R, Sambuy Y, et al. Permeability characteristics of parental and clonal human intestinal Caco-2 cell lines differentiated in Serum-supplemented and serum-free media. Toxicology in Vitro. 2003;17:761-767. doi:10.1016/S0887-2333(03)00095-X
38. Cheol-Hee C, Joon-Ho K, Sang-H K, et al. Reversal of P-glycoprotein-mediated MDR by 5,7,3',4',5'-pentamethoxyflavone and SAR. Biochem Biophys Res Commun. 2004;320(3):672. doi:10.1016/j.bbrc.2004.06.020

39. Artursson P, Palm K, Luthman K, et al. Caco-2 monolayers in experimental and theoretical predictions of drug transport. Adv Drug Deliv Rev. 2012;64(12):280-289. doi:10.1016/j.addr.2012.09.005

40. Xu Y, Zheng Y, Wu L, et al. Novel solid lipid nanoparticle with endosomal escape function for oral delivery of insulin. ACS Appl Mater Interfaces. 2018;10(11):9315-9324. doi:10.1021/acsami.8b00507

41. He Z, Santos JL, Tian H, et al. Scalable fabrication of size-controlled chitosan nanoparticles for oral delivery of insulin. Biomaterials. 2017;130:28-41. doi:10.1016/j.biomaterials.2017.03.028

42. Xia D, He H, Wang Y, et al. Ultrafast glucose-responsive, high loading capacity erythrocyte to self-regulate the release of insulin. Acta Biomater. 2018;69:301-312. doi:10.1016/j.actbio.2018.01.029

43. Bai X, Kong M, Xia G, et al. Systematic investigation of fabrication conditions of nanocarrier based on carboxymethyl chitosan for sustained release of insulin. Int J Biol Macromol. 2017;102:468-474. doi:10.1016/j.ijbiomac.2017.03.181

44. Barbari GR, Dorkoosh FA, Amini M, et al. A novel nanoemulsion-based method to produce ultrasmall, water-dispersible nanoparticles from chitosan, surface modified with cell-penetrating peptide for oral delivery of proteins and peptides. Int $J$ Nanomed. 2017;12:3471-3483. doi:10.2147/IJN.S116063

45. Heni W, Vonna L, Haidara H. Experimental characterization of the nanoparticle size effect on the mechanical stability of nanoparticle-based coatings. Nano Lett. 2015;15(1):442-449. doi: $10.1021 / \mathrm{nl} 503768 \mathrm{r}$

46. Xia Y, Guo M, Xu T, et al. siRNA-loaded selenium nanoparticle modified with hyaluronic acid for enhanced hepatocellular carcinoma therapy. Int $J$ Nanomed. 2018;13:1539-1552. doi:10.2147/IJN. S157519

47. Maisel K, Ensign L, Reddy M, et al. Effect of surface chemistry on nanoparticle interaction with gastrointestinal mucus and distribution in the gastrointestinal tract following oral and rectal administration in the mouse. J Controlled Release. 2015;197:48-57. doi:10.1016/j. jconrel.2014.10.026

48. Zheng Y, Wu J, Shan W, et al. Correction to multifunctional nanoparticles enable efficient oral delivery of biomacromolecules via improving payload stability and regulating the transcytosis pathway. ACS Appl Mater Interfaces. 2019;11(19):48-52.

49. Jin Y, Song Y, Zhu X, et al. Goblet cell-targeting nanoparticles for oral insulin delivery and the influence of mucus on insulin transport. Biomaterials. 2012;33(5):1573-1582. doi:10.1016/j.biomaterials. 2011.10.075

50. Sung HW, Sonaje K, Liao ZX, et al. pH-responsive nanoparticles shelled with chitosan for oral delivery of insulin: from mechanism to therapeutic applications. Acc Chem Res. 2012;45(4):619-629. doi:10.1021/ar200234q

51. Sonaje K, Lin KJ, Tseng MT, et al. Effects of chitosan-nanoparticlemediated tight junction opening on the oral absorption of endotoxins. Biomaterials. 2011;32(33):8712-8721. doi:10.1016/j.biomaterials. 2011.07.086

52. Aguirre TA, Teijeiro-Osorio D, Rosa M, et al. Current status of selected oral peptide technologies in advanced preclinical development and in clinical trials. Adv Drug Deliv Rev. 2016;106(Pt B):223-241. doi:10.1016/j.addr.2016.02.004

53. Matteucci E, Giampietro O, Covolan V, et al. Insulin administration: present strategies and future directions for a noninvasive (possibly more physiological) delivery. Drug Des Devel Ther. 2015;9:3109-3118. doi:10.2147/DDDT.S79322

54. Jin X, Asghar S, Zhang M, et al. N-acetylcysteine modified hyaluronic acid-paclitaxel conjugate for efficient oral chemotherapy through mucosal bioadhesion ability. Colloids Surf $B$ Biointerfaces. 2018;172:655-664. doi:10.1016/j.colsurfb.2018.09.025 


\section{Publish your work in this journal}

The International Journal of Nanomedicine is an international, peerreviewed journal focusing on the application of nanotechnology in diagnostics, therapeutics, and drug delivery systems throughout the biomedical field. This journal is indexed on PubMed Central, MedLine, CAS, SciSearch ${ }^{\circledR}$, Current Contents ${ }^{\circledR} /$ Clinical Medicine,
Journal Citation Reports/Science Edition, EMBase, Scopus and the Elsevier Bibliographic databases. The manuscript management system is completely online and includes a very quick and fair peer-review system, which is all easy to use. Visit http://www.dovepress.com/ testimonials.php to read real quotes from published authors. 